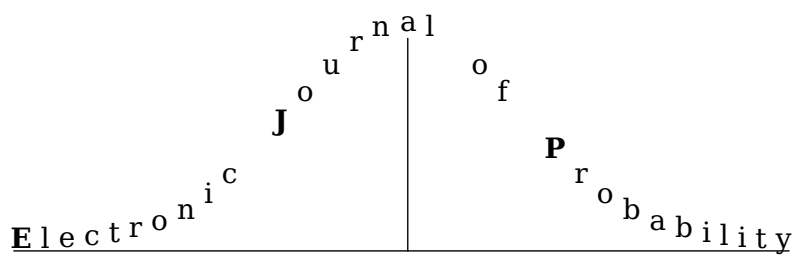

Electron. J. Probab. 26 (2021), article no. 136, 1-24.

ISSN: 1083-6489 https://doi.org/10.1214/21-EJP694

\title{
Projections of martingales in enlargements of Brownian filtrations under Jacod's equivalence hypothesis*
}

\author{
Pavel V. Gapeev ${ }^{\dagger} \quad$ Monique Jeanblanc $^{\ddagger} \quad$ Dongli Wu $^{\S}$
}

\begin{abstract}
We consider the initial and progressive enlargements of a Brownian filtration with a random time, that is, a strictly positive random variable. We assume Jacod's equivalence hypothesis, that is, the existence of a strictly positive conditional density for the random time with respect to the Brownian filtration. Then, starting with the predictable integral representation of a martingale in the initially enlarged Brownian filtration, we derive explicit expressions for the components which appear in the predictable integral representations for the optional projections of the martingale on the progressively enlarged filtration and on the Brownian filtration. We also provide similar results for the optional projection of a martingale in the progressively enlarged filtration on the Brownian filtration.
\end{abstract}

Keywords: Brownian motion; conditional probability density; Jacod's equivalence hypothesis; initial and progressive enlargements of filtrations; predictable (martingale) representation property; changes of probability measures.

MSC2020 subject classifications: Primary 60G44; 60J65; 60G40, Secondary 60G35; 60H10; 91G40.

Submitted to EJP on September 15, 2019, final version accepted on August 27, 2021.

\section{Introduction}

In this paper, we consider the initial (resp. progressive) enlargement of a Brownian filtration $\mathbb{F}$ (called hereafter the reference filtration) with a strictly positive absolutely continuous random variable $\tau$ (called hereafter a random time), denoted by $\mathbb{F}^{(\tau)}$ (resp. $\mathbb{G}$ ). We assume Jacod's equivalence hypothesis introduced in [2] and [13] (see Section 3

\footnotetext{
*This research benefited from the support of the 'Chaire Marchés en Mutation', French Banking Federation and ILB, Labex ANR 11-LABX-0019.

${ }^{\dagger}$ London School of Economics, Department of Mathematics, Houghton Street, London WC2A 2AE, United Kingdom. E-mail: p.v.gapeev@lse.ac.uk

${ }^{\ddagger}$ Université Paris-Saclay, CNRS, Univ Evry, Laboratoire de Mathématiques et Modélisation d’Evry, 91037, Evry, France. E-mail: monique.jeanblanc@univ-evry.fr

${ }^{\S}$ CCB Fintech. E-mail: wudongli. sh@ccbft.com
} 
Projections of martingales in enlargements of Brownian filtrations

below for details), which implies that there exists an $\mathbb{F}^{(\tau)}$-martingale enjoying the predictable representation property with respect to $\mathbb{F}^{(\tau)}$ (see Theorem 4.6 in [3]) and a pair of $\mathbb{G}$-martingales enjoying the predictable representation property with respect to $\mathbb{G}$ (see Theorem 6.4 in [18]). We study the relationship between the representation of martingales in the initially (resp. progressively) enlarged filtration and the various optional projections. We refer the reader to the monograph [1] for results on enlargements of filtrations. An application of our results is presented in [4] for the study of the characteristics of semimartingales and their optional projections. Our results will be useful to compare the optimal strategies of investors having different information flows, and to investigate optimal stopping problems in different filtrations. Note that the arguments developed in the paper can be extended to the case of models driven by marked point processes that we study in [12].

The paper is organised as follows. In Section 2, we recall standard definitions of projections and dual projections as well as other results on stochastic analysis that we use in the paper. In Section 3, we give some basic definitions and results related to the initial and progressive enlargements of a Brownian filtration under Jacod's equivalence hypothesis. In Section 4, we recall that the predictable representation property holds with respect to explicit martingales in the filtrations involved, and prove that any $\mathbb{F}^{(\tau)}$. martingale is continuous. We determine the multiplicity (or spanning number) of these filtrations (see [7] and [9] for the description of this concept). In Section 5, we consider the optional projections of an $\mathbb{F}^{(\tau)}$-martingale on the filtrations $\mathbb{G}$ and $\mathbb{F}$. In particular, we derive explicit expressions for the components in the integral representations of these optional projections in terms of the original $\mathbb{F}^{(\tau)}$-martingale and the components in its representation as a stochastic integral and give analogous results in the case of the $\mathbb{F}$-optional projection of a $\mathbb{G}$-martingale. In Section 6, we consider the optional projections of a positive $\mathbb{F}^{(\tau)}$-martingale on $\mathbb{G}$ and $\mathbb{F}$ and the $\mathbb{F}$-optional projection of a positive $\mathbb{G}$-martingale. We describe the set of equivalent martingale measures in the associated extension of the Black-Merton-Scholes model enhanced with the random time $\tau$. In particular, we show that the set of equivalent martingale measures in the model with the progressively enlarged filtration $\mathbb{G}$ is essentially larger than the one obtained by means of the optional projections on $\mathbb{G}$ of the Radon-Nikodym densities in the model with the initially enlarged filtration $\mathbb{F}^{(\tau)}$.

\section{Preliminary definitions and results}

For the ease of the reader, we recall some basic definitions and notation on stochastic analysis. We assume that $(\Omega, \mathcal{G}, \mathbb{P})$ is a probability space endowed with a filtration $\mathbb{H}$ satisfying the usual hypotheses of completeness and right continuity. For any pair $X$, $Y$ of $\mathbb{H}$-semimartingales, we denote by $\langle X, Y\rangle^{\mathbb{H}}$ the associated predictable quadratic covariation, when it exists (see page 210, line 6 in [14]), and, for simplicity, $\langle X, X\rangle^{\mathbb{H}}$ is denoted by $\langle X\rangle^{\mathrm{H}}$.

We start with the definitions of predictable and optional projections as well as dual predictable and optional projections (see Chapter V, Theorem 5.1 and 5.2, pages 135-136 in [14] for the definition and Sections 1-2 in the same chapter for more information).

Definition 2.1. Let $X=\left(X_{t}\right)_{t \geq 0}$ be a measurable process such that, for any H-stopping time $\vartheta$, the random variable $X_{\vartheta} \mathbb{1}_{\{\vartheta<\infty\}}$ is $\sigma$-integrable with respect to $\mathcal{H}_{\vartheta}$. Then there exists a unique $\mathrm{H}$-optional process ${ }^{o} X^{\mathrm{H}}=\left({ }^{o} X_{t}^{\mathbb{H}}\right)_{t \geq 0}$ satisfying, for any $\mathrm{H}$-stopping time $\vartheta$,

$$
\mathbb{E}\left[X_{\vartheta} \mathbb{1}_{\{\vartheta<\infty\}} \mid \mathcal{H}_{\vartheta}\right]={ }^{o} X_{\vartheta}^{\mathbb{H}} \mathbb{1}_{\{\vartheta<\infty\}} .
$$

The process ${ }^{o} X^{\mathrm{H}}$ is called the $\mathrm{H}$-optional projection of $X$. 
Remark 2.2. Note that, if $X$ is a càdlàg process, then so is the process ${ }^{\circ} X^{\mathrm{H}}$ (cf. Theorem VI-47 in [8]). Moreover, if $X$ is a càdlàg process and $H$ satisfies usual conditions, then the process $\left(\mathbb{E}\left[X_{t} \mid \mathcal{H}_{t}\right]\right)_{t \geq 0}$ is a càdlàg process too. Finally, since ${ }^{o} X^{\mathrm{H}}$ is a modification of $\left(\mathbb{E}\left[X_{t} \mid \mathcal{H}_{t}\right]\right)_{t \geq 0}$ and these processes are both càdlàg, then they are indistinguishable (cf. Chapter II, Definition 2.45, pages 55-56 in [14]). In particular, if $X$ is a IK-martingale and $\mathbb{H} \subset \mathbb{K}$, then the optional projection ${ }^{\circ} X^{\mathrm{H}}$ is an $\mathbb{H}$-martingale.

Definition 2.3. Let $X=\left(X_{t}\right)_{t \geq 0}$ be a measurable process such that, for any predictable $\mathbb{H}$-stopping time $\vartheta$, the random variable $X_{\vartheta} \mathbb{1}_{\{\vartheta<\infty\}}$ is $\sigma$-integrable with respect to $\mathcal{H}_{\vartheta-}$. Then there exists a unique $\mathrm{H}$-predictable process ${ }^{p} X^{\mathrm{H}}=\left({ }^{p} X_{t}^{\mathrm{H}}\right)_{t \geq 0}$ satisfying, for any $\mathrm{H}$-predictable stopping time $\vartheta$,

$$
\mathbb{E}\left[X_{\vartheta} \mathbb{1}_{\{\vartheta<\infty\}} \mid \mathcal{H}_{\vartheta-}\right]={ }^{p} X_{\vartheta}^{\mathbb{H}} \mathbb{1}_{\{\vartheta<\infty\}} .
$$

The process ${ }^{p} X^{\mathbb{H}}$ is called the $\mathbb{H}$-predictable projection of $X$.

Let $V=\left(V_{t}\right)_{t \geq 0}$ be a process of locally integrable variation. Then, there exists a unique $\mathbb{H}$-optional process of locally integrable variation $V^{o, H}$, called the $\mathbb{H}$-dual optional projection of $V$, such that

$$
\mathbb{E}\left[\int_{[0, \infty)} U_{s} d V_{s}\right]=\mathbb{E}\left[\int_{[0, \infty)} U_{s} d V_{s}^{o, H}\right]
$$

for any H-optional process $U=\left(U_{t}\right)_{t \geq 0}$ satisfying

$$
\mathbb{E}\left[\int_{[0, \infty)}\left|U_{s}\right| d\left|V_{s}\right|\right]<\infty
$$

Similarly, there exists a unique $\mathrm{H}$-predictable process $V^{p, \mathrm{H}}$ of locally integrable variation, called the $\mathbb{H}$-dual predictable projection of $V$, such that

$$
\mathbb{E}\left[\int_{[0, \infty)} U_{s} d V_{s}\right]=\mathbb{E}\left[\int_{[0, \infty)} U_{s} d V_{s}^{p, \mathbb{H}}\right]
$$

for any H-predictable process $U=\left(U_{t}\right)_{t \geq 0}$ satisfying (2.1).

Note that, if $\mathbb{H}$ is a continuous filtration ${ }^{1}$, then $\mathbb{H}$-dual optional and $\mathbb{H}$-dual predictable projections are equal. Indeed, the optional process $V^{o, H}$ is predictable (see, e.g., Chapter IV, Corollary 5.7, page 173 in [24]) and, since $\left(V^{o, \mathbb{H}}\right)^{p, H}=V^{p, H}$, the result follows.

For two processes $X=\left(X_{t}\right)_{t \geq 0}$ and $Y=\left(Y_{t}\right)_{t \geq 0}$, we write $X=Y$, when they are indistinguishable.

The notation $\theta \cdot X$ is used for the stochastic integral with respect to a semimartingale $X$, that is, we set

$$
(\theta \cdot X)_{t}=\int_{0}^{t} \theta_{s} d X_{s}, \forall t \geq 0
$$

when it exists.

We shall make use of the multiplicative decomposition of càdlàg $\mathbb{H}$-supermartingales (see Chapter II, Theorem 8.21, page 138 in [16] for the multiplicative decomposition of strictly positive special semimartingales or Proposition 1.32, page 15 in [1] for strictly positive supermartingales), which states that a strictly positive càdlàg $\mathrm{H}$-semimartingale $Y=\left(Y_{t}\right)_{t \geq 0}$ admits a unique decomposition as

$$
Y_{t}=N_{t} D_{t}, \forall t \geq 0,
$$

\footnotetext{
${ }^{1} \mathrm{~A}$ continuous filtration is a filtration $\mathbb{H}$ such that any $\mathrm{H}$-martingale is continuous. The basic example is the Brownian filtration.
} 
where $N=\left(N_{t}\right)_{t>0}$ is an H-local martingale, with $N_{0}=1$ and $D=\left(D_{t}\right)_{t>0}$ an $\mathbb{H}$ predictable process with locally finite variation. In Chapter II of [16], explicit expressions for $N$ and $D$ can be found in the case of semimartingales, and it is easy to check with these formulae that the process $D$ is decreasing when $Y$ is a supermartingale. In our setting, we shall present in (4.20) and (4.21) an explicit computation.

We introduce the stochastic exponential of a càdlàg H-local martingale $X$ which is the $\mathbb{H}$-local martingale $\mathcal{E}(X)=\left(\mathcal{E}(X)_{t}\right)_{t \geq 0}$ defined by

$$
\mathcal{E}(X)_{t}=e^{X_{t}-X_{0}-\frac{1}{2}\left\langle X^{c}, X^{c}\right\rangle_{t}} \prod_{0<s \leq t}\left(1+\Delta X_{s}\right) e^{-\Delta X_{s}}, \forall t \geq 0,
$$

where $X^{c}$ is the continuous $\mathrm{H}$-martingale part of $X$, and $\Delta X_{t}=X_{t}-X_{t-}$ (see, e.g., Chapter IX, Theorem 9.39, page 248 in [14] or Chapter I, Formula 4.64, page 59 in [16]). The process $\mathcal{E}(X)$, called the Doléans-Dade exponential of $X$, is the unique solution of the stochastic differential equation (see Chapter I, Formula 4.59, page 59 in [16] or Chapter IX, Theorem 9.39, page 248 in [14])

$$
d Z_{t}=Z_{t-} d X_{t}, \quad Z_{0}=1 .
$$

For instance, in a Brownian filtration $\mathbb{F}$, for an $\mathbb{F}$-optional process $\theta=\left(\theta_{t}\right)_{t \geq 0}$ satisfying $\int_{0}^{t} \theta_{s}^{2} d s<\infty$ (P-a.s.), for all $t \geq 0$, the process $Z=\mathcal{E}(\theta \cdot W)$ is the $\mathbb{F}$-local martingale solution of the equation

$$
d Z_{t}=Z_{t} \theta_{t} d W_{t}, \quad Z_{0}=1 .
$$

The following proposition is a particular case of the result of Chapter II, Theorem 8.21, page 138 in [16], suitable for our purposes.

Proposition 2.4. Let $M=\left(M_{t}\right)_{t \geq 0}$ be an $\mathbb{H}$-martingale and $X=1+(\theta \cdot M)$, where $\theta=\left(\theta_{t}\right)_{t \geq 0}$ is an $\mathbb{H}$-predictable and locally bounded process. We assume that $X$ and $X_{-}$ take their values in $(0, \infty)$. Then, the process $X$ is a stochastic exponential martingale, that is, there exists an $\mathbb{H}$-predictable locally bounded process $\psi$ such that equality $X=\mathcal{E}(\psi \cdot M)$ holds.

A probability measure $\mathbb{Q}$ is said to be locally equivalent to $\mathbb{P}$ on the filtration $\mathbb{H}$ if there exists a strictly positive $\mathrm{H}$-martingale $L=\left(L_{t}\right)_{t \geq 0}$ such that

$$
\left.\frac{d \mathbb{Q}}{d \mathbb{P}}\right|_{\mathcal{H}_{t}}=L_{t}, \forall t \geq 0 .
$$

The martingale $L$ is called the Radon-Nikodym density of $\mathbb{Q}$ with respect to $\mathbb{P}$. The "locally" terminology is needed, since, in Section 6 of our paper, as in [3], we cannot define the new probability measure $\mathbb{Q}$ on $\mathcal{H}_{\infty}$.

As usual, $\mathcal{B}\left(\mathbb{R}^{+}\right)$is the Borel $\sigma$-algebra on $\mathbb{R}^{+}$, and $\mathcal{P}(\mathbb{H})$ (resp. $\mathcal{O}(\mathbb{H})$ ) denotes the predictable (resp. optional) $\sigma$-algebra associated with $\mathbb{H}$.

\section{Jacod's equivalence hypothesis}

In the whole paper, we work on a probability space $(\Omega, \mathcal{G}, \mathbb{P})$ which supports a standard Brownian motion $W=\left(W_{t}\right)_{t \geq 0}$ with a continuous and completed natural filtration $\mathbb{F}=\left(\mathcal{F}_{t}\right)_{t \geq 0}$ and a strictly positive random variable $\tau$. We assume that the law of $\tau$ has the support $\mathbb{R}^{+}$and admits a density $g$ with respect to Lebesgue's measure. Note that the inclusion $\mathcal{F}_{\infty} \subset \mathcal{G}$ holds and, in general, this inclusion is strict. We recall that any $\mathbb{F}$-martingale is continuous.

We assume, as in [3] and [13], that Jacod's equivalence hypothesis holds, that is, for each $t \geq 0$, the regular conditional distribution of $\tau$ given $\mathcal{F}_{t}$ is equivalent to the law of $\tau$ :

$$
\mathbb{P}\left(\tau \in \cdot \mid \mathcal{F}_{t}\right) \sim \mathbb{P}(\tau \in \cdot), \forall t \geq 0(\mathbb{P} \text {-a.s. }) .
$$


In our model, due to the existence of a density for $\tau$, and the fact that $\mathbb{F}$ is a continuous filtration, this assumption implies (see Lemma 2.2 in [3]) that there exists a family of strictly positive processes $p(u)=\left(p_{t}(u)\right)_{t \geq 0}$ such that the function $(\omega, t, u) \mapsto p_{t}(u ; \omega)$ is $\mathcal{O}(\mathbb{F}) \otimes \mathcal{B}\left(\mathbb{R}^{+}\right)$-measurable, and that, for each $u \geq 0$, the process $p(u)$ is an $\mathbb{F}$-martingale. Furthermore, for any bounded Borel function $f$, the following equality holds

$$
\mathbb{E}\left[f(\tau) \mid \mathcal{F}_{t}\right]=\int_{0}^{\infty} f(u) p_{t}(u) g(u) d u, \forall t \geq 0 \text { (P-a.s.) } .
$$

The expression in (3.1) implies that the following equality holds

$$
\mathbb{P}\left(\tau>s \mid \mathcal{F}_{t}\right)=\int_{s}^{\infty} p_{t}(u) g(u) d u, \forall t, s \geq 0(\mathbb{P} \text {-a.s. })
$$

so that, from the strict positivity of $\tau$, the equality

$$
\int_{0}^{\infty} p_{t}(u) g(u) d u=1, \forall t \geq 0(\mathbb{P} \text {-a.s. }),
$$

is satisfied, and $p_{0}(u)=1$, for each $u \geq 0$.

Proposition 3.1. For each $u \geq 0$, there exists an $\mathbb{F}$-predictable process $\varphi(u)=\left(\varphi_{t}(u)\right)_{t \geq 0}$ such that the strictly positive $\mathbb{F}$-martingale $p(u)$ admits the representation in the form of the Doléans-Dade stochastic exponential

$$
p_{t}(u)=\mathcal{E}(\varphi(u) \cdot W)_{t}=\exp \left(\int_{0}^{t} \varphi_{s}(u) d W_{s}-\frac{1}{2} \int_{0}^{t} \varphi_{s}^{2}(u) d s\right), \forall t \geq 0,
$$

or, equivalently, $p(u)$ satisfies the stochastic differential equation

$$
d p_{t}(u)=p_{t}(u) \varphi_{t}(u) d W_{t}, \quad p_{0}(u)=1
$$

Proof. This result is an immediate application of the predictable representation theorem in a Brownian filtration (see, e.g., Chapter V, Theorem 3.5, page 201 in [24]) which states that, for each $u \geq 0$, there exists an $\mathbb{F}$-predictable and locally bounded process $\theta(u)=\left(\theta_{t}(u)\right)_{t \geq 0}$ such that $p(u)=1+(\theta(u) \cdot W)$ holds. Then, it follows from Proposition 2.4 that the representations in (3.2) and (3.3) hold with $\varphi(u)=\theta(u) / p(u$ ), for each $u \geq 0$ (see also Chapter 5, Theorem 5.9, page 181 in [22]). It is shown in [26] that $u \mapsto \varphi_{t}(u)$ is a Borel function on $[0, \infty)$, for all $t \geq 0$ (P-a.s.).

Let $H^{\mathbb{G}}=\left(H_{t}^{\mathbb{G}}\right)_{t \geq 0}$ be the indicator default process defined by $H_{t}^{\mathbb{G}}:=\mathbb{1}_{\{\tau \leq t\}}$, for each $t \geq 0$. In the credit risk theory, $\tau$ usually denotes the time when a default occurs. Moreover, since $H^{\mathbb{G}}$ is a càdlàg process, we can introduce the $\mathbb{F}$-supermartingale $G=$ $\left(G_{t}\right)_{t \geq 0}$ defined by $G={ }^{o, F}\left(1-H^{\mathbb{G}}\right)$, that is, the $\mathbb{F}$-optional projection of $1-H^{\mathbb{G}}$ satisfying the property

$$
G_{t}=\mathbb{P}\left(\tau>t \mid \mathcal{F}_{t}\right), \forall t \geq 0(\mathbb{P} \text {-a.s. })
$$

which, according to the equality (3.1), can also be written as

$$
G_{t}=\int_{t}^{\infty} p_{t}(u) g(u) d u, \forall t \geq 0 \text { (P-a.s.). }
$$

Note that $G$ is strictly positive and continuous and that, from the strict positivity of $\tau$, one has $G_{0}=1$. The $\mathbb{F}$-supermartingale $G$ is called the conditional survival process or the Azéma supermartingale of the random time $\tau$. 


\section{Enlargement of filtrations and martingales}

The aim of the paper is to explicitly compute the components in the integral representations of the optional projections of the $\mathbb{F}^{(\tau)}$-martingales and of the $\mathbb{G}$-martingales. In this section, we recall some well known results. We give the form of the $\mathbb{F}^{(\tau)}$. semimartingale decomposition and $\mathbb{G}$-semimartingale decomposition of $W$ as well as the $\mathbb{G}$-semimartingale decomposition of $H^{\mathbb{G}}$. We underline that the martingale part $W^{(\tau)}$ of the $\mathbb{F}^{(\tau)}$-semimartingale decomposition of $W$ enjoys the $\mathbb{F}^{(\tau)}$-predictable representation property, while the pair $\left(W^{\mathbb{G}}, M^{\mathbb{G}}\right)$ of the martingale parts of the $\mathbb{G}$-semimartingale decompositions of $W$ and $H^{\mathbb{G}}$ enjoys the $\mathbb{G}$-predictable representation property (see below in (4.8) and (4.12) the explicit form of this pair).

\subsection{The initially enlarged filtration}

As in the introduction, let us denote by $\mathbb{F}^{(\tau)}=\left(\mathcal{F}_{t}^{(\tau)}\right)_{t \geq 0}=\left(\mathcal{F}_{t} \vee \sigma(\tau)\right)_{t \geq 0}$ the initial enlargement of the filtration $\mathbb{F}$ with the random time $\tau$. We recall that, under Jacod's equivalence hypothesis, any $\mathbb{F}$-local martingale is an $\mathbb{F}^{(\tau)}$-special semimartingale (see, e.g., Theorem 2.1 in [15] or Proposition 5.30, page 116 in [1]). Note that, according to Proposition 3.3 in [3], the filtration $\mathbb{F}^{(\tau)}$ is right-continuous. We also recall the following important result (see, e.g., Proposition 4.22, page 86 in [1] or Proposition 2.7, part (i) in [6]).

Lemma 4.1. For any $t \geq 0$ fixed, any $\mathcal{F}_{t}^{(\tau)}$-measurable random variable is of the form $Y_{t}(\omega, \tau(\omega))$, for some $\mathcal{F}_{t} \otimes \mathcal{B}\left(\mathbb{R}^{+}\right)$-measurable function $(\omega, u) \mapsto Y_{t}(\omega, u)$. In particular, any $\mathcal{F}_{0}^{(\tau)}$-measurable random variable is a Borel function of $\tau$.

In the light of the previous lemma, we denote by $Y(\tau)=\left(Y_{t}(\tau)\right)_{t \geq 0}$ the processes adapted with respect to the filtration $\mathbb{F}^{(\tau)}$. Recall that any $\mathbb{F}^{(\tau)}$-predictable process can be represented in the form $\left(Y_{t}(\omega, \tau(\omega)), t \geq 0\right)$, where the mapping $(\omega, t, u) \mapsto$ $Y_{t}(\omega, u)$ defined on $\Omega \times \mathbb{R}^{+} \times \mathbb{R}^{+}$and valued in $\mathbb{R}$, is $\mathcal{P}(\mathbb{F}) \otimes \mathcal{B}\left(\mathbb{R}^{+}\right)$-measurable (see, e.g., Proposition 4.22, page 86 in [1]). As an immediate consequence of Jacod's equivalence hypothesis, we observe that, for each $t \geq 0$, if the $\mathcal{F}_{t}^{(\tau)}$-measurable random variable $Y_{t}(\tau)$ is integrable, then the following representation holds

$$
\mathbb{E}\left[Y_{t}(\tau) \mid \mathcal{F}_{t}\right]=\int_{0}^{\infty} Y_{t}(u) p_{t}(u) g(u) d u, \forall t \geq 0,
$$

(see, e.g., Proposition 4.18 (b), page 85 in [1]). Theorem 2.1 in [15] applies to prove that the process $W(\tau)=\left(W_{t}(\tau)\right)_{t \geq 0}$ defined by

$$
W_{t}(\tau)=W_{t}-\left.\int_{0}^{t} \frac{d\langle W, p(u)\rangle_{s}^{\mathbb{F}}}{p_{s}(u)}\right|_{u=\tau}=W_{t}-\int_{0}^{t} \varphi_{s}(\tau) d s, \forall t \geq 0,
$$

is an $\mathbb{F}^{(\tau)}$-standard Brownian motion, where the second equality follows from (3.3).

Remark 4.2. Note that, because of integrability reasons, the fact that $W$ is an $\mathbb{F}^{(\tau)}$ semimartingale does not imply that any $\mathbb{F}$-martingale is an $\mathbb{F}^{(\tau)}$-semimartingale ${ }^{2}$. However, under Jacod's equivalence hypothesis, for any $\mathbb{F}$-martingale $X$, the process $X(\tau)$

${ }^{2}$ Indeed, if $X$ is any $\mathbb{F}$-martingale, it admits the representation in the form

$$
X_{t}=X_{0}+\int_{0}^{t} \theta_{s} d W_{s}, \forall t \geq 0,
$$

for some suitable process $\theta=\left(\theta_{t}\right)_{t \geq 0}$. One can think that $X$ is an $\mathbb{F}^{(\tau)}$-semimartingale is an immediate consequence of the equality (4.2) writing

$$
X_{t}=X_{0}+\int_{0}^{t} \theta_{s} d W_{s}(\tau)+\int_{0}^{t} \varphi_{s}(\tau) \theta_{s} d s, \forall t \geq 0 .
$$


given by

$$
X_{t}(\tau)=X_{t}-\left.\int_{0}^{t} \frac{d\langle X, p(u)\rangle_{s}^{\mathbb{F}}}{p_{s}(u)}\right|_{u=\tau}, \forall t \geq 0,
$$

is an $\mathbb{F}^{(\tau)}$-martingale (see, e.g., Theorem 2.1 in [15] or Proposition 4.25, page 88 in [1]).

We recall that the $\mathbb{F}^{(\tau)}$-standard Brownian motion $W(\tau)$ enjoys the $\mathbb{F}^{(\tau)}$-predictable representation property (see, e.g., [3]). Therefore, any $\mathbb{F}^{(\tau)}$-martingale $Y(\tau)$ is continuous and admits the representation

$$
Y_{t}(\tau)=Y_{0}(\tau)+\int_{0}^{t} y_{s}(\tau) d W_{s}(\tau), \forall t \geq 0,
$$

where $u \mapsto Y_{0}(u)$ is $\mathcal{B}\left(\mathbb{R}^{+}\right)$-measurable and the map $(\omega, t, u) \mapsto y_{t}(\omega, u)$ is $\mathcal{P}(\mathbb{F}) \otimes \mathcal{B}\left(\mathbb{R}^{+}\right)$measurable.

\subsection{The progressively enlarged filtration}

We denote by $\mathbb{G}=\left(\mathcal{G}_{t}\right)_{t \geq 0}$ the progressive enlargement of $\mathbb{F}$ with $\tau$, that is,

$$
\mathcal{G}_{t}=\cap_{s>t} \mathcal{F}_{s} \vee \sigma(\tau \wedge s), \forall t \geq 0 .
$$

Note that $\tau$ is a $\mathbb{G}$-stopping time and that, according to the hypothesis that the positive random variable $\tau$ has a positive density with support $\mathbb{R}^{+}$, the $\sigma$-algebra $\mathcal{G}_{0}$ is trivial, so that the initial value of a $\mathbb{G}$-adapted process is a deterministic one. Observe that, under Jacod's equivalence hypothesis, any $\mathbb{F}$-martingale is a $\mathbb{G}$-semimartingale (see, e.g., Proposition 5.30, page 116 in [1] or Theorem 3.1 in [19]), and thus, a special semimartingale (see, e.g., Chapter VI, Theorem 4, page 367 in [23]). We also recall for the ease of the reader a result which follows from Lemma 3.1 in [11] (see also Lemma 7.4.1.1 in [17]). Lemma 3.1 in [11] follows by the fact that, for all $t \geq 0$, any $\mathcal{G}_{t}$-measurable random variable is equal to an $\mathcal{F}_{t}$-measurable random variable on the set $\{t<\tau\}$.

Lemma 4.3. For any integrable random variable $Z \in \mathcal{F}_{T}$, the following equality holds

$$
\mathbb{E}\left[Z \mathbb{1}_{\{T<\tau\}} \mid \mathcal{G}_{t}\right]=\mathbb{1}_{\{t<\tau\}} \frac{\mathbb{E}\left[Z G_{T} \mid \mathcal{F}_{t}\right]}{G_{t}}, \forall t \geq 0,
$$

where $G$ is the Azéma supermartingale defined by the expression in (3.4).

We further indicate with the superscript $\mathbb{G}$ the processes which are $\mathbb{G}$-adapted, as $Y^{\mathbb{G}}$, while we do not use the superscript $\mathbb{F}$ to denote the processes which are $\mathbb{F}$-adapted, as $Y, Y^{0}$, or $y(u)$.

Since $\mathbb{G}$ coincides with $\mathbb{F}$ on $\{t<\tau\}$ and with $\mathbb{F}^{(\tau)}$ on $\{\tau \leq t\}$, for each $t \geq 0$, one has the following lemma (see Proposition 2.8 (i) in [6]):

Lemma 4.4. For any fixed $t \geq 0$, the random variable $Y_{t}^{\mathbb{G}}$ is $\mathcal{G}_{t}$-measurable if and only if it is of the form

$$
Y_{t}^{\mathbb{G}}(\omega)=y_{t}(\omega) \mathbb{1}_{\{t<\tau(\omega)\}}+y_{t}^{1}(\omega, \tau(\omega)) \mathbb{1}_{\{\tau(\omega) \leq t\}},
$$

for some $\mathcal{F}_{t}$-measurable random variable $y_{t}$ and some $\mathcal{F}_{t} \otimes \mathcal{B}\left(\mathbb{R}^{+}\right)$-measurable function $(\omega, u) \mapsto y_{t}^{1}(\omega, u)$.

However, it may happen that $\theta$ is such that the process

$$
\int_{0}^{t} \varphi_{s}(\tau) \theta_{s} d s, \forall t \geq 0
$$

is not defined (see Theorem 3 and the example given in Corollaire 3.1 in [21]), and $X$ is not an $\mathbb{F}^{(\tau)}$. semimartingale. 
This simple property can be extended (with care) to the case of processes as follows. Namely, under Jacod's equivalence hypothesis, if the process $Y^{\mathbb{G}}$ is $\mathbb{G}$-optional, then, according to Theorem 6.9 in [25], it can be represented as

$$
Y_{t}^{\mathbb{G}}=\mathbb{1}_{\{\tau>t\}} Y_{t}^{0}+\mathbb{1}_{\{\tau \leq t\}} Y_{t}^{1}(\tau), \forall t \geq 0
$$

where the process $Y^{0}$ is $\mathbb{F}$-optional and the map $(\omega, t, u) \mapsto Y_{t}^{1}(\omega, u)$ is $\mathcal{O}(\mathbb{F}) \otimes \mathcal{B}\left(\mathbb{R}^{+}\right)$measurable (we shall say that $Y^{1}$ is an $\mathcal{O}(\mathbb{F}) \otimes \mathcal{B}\left(\mathbb{R}^{+}\right)$-measurable process). We further call $Y^{0}$ the $\mathbb{F}$-optional reduction of $Y^{\mathbb{G}}$, which is uniquely defined in our setting due to the strict positivity of $G$ (see the arguments below). The uniqueness of the second part $Y_{t}(u)$ is valid only for $t \geq u$. This issue does not matter, since these processes appear only in the part in which the uniqueness holds.

For the ease of the reader, we recall the proof of this as it is done in Proposition 5.25 (b), page 113 in [1]. From (4.5), one has $Y_{t}^{\mathbb{G}} \mathbb{1}_{\{t<\tau\}}=Y_{t}^{0} \mathbb{1}_{\{t<\tau\}}$, for each $t \geq 0$, therefore, taking conditional expectation of both sides with respect to $\mathcal{F}_{t}$, and using the fact that $G$ is strictly positive

$$
Y_{t}^{0}=\frac{1}{G_{t}} \mathbb{E}\left[Y_{t}^{\mathbb{G}} \mathbb{1}_{\{t<\tau\}} \mid \mathcal{F}_{t}\right], \forall t \geq 0
$$

Since $Y_{t}^{\mathrm{G}}$ is defined as the optional projection (see Remark 2.2) of $Y_{t}(\tau)$,

$$
\mathbb{E}\left[Y_{t}^{\mathbb{G}} \mathbb{1}_{\{t<\tau\}} \mid \mathcal{F}_{t}\right] \stackrel{\mathrm{TP}}{=} \mathbb{E}\left[Y_{t}(\tau) \mathbb{1}_{\{t<\tau\}} \mid \mathcal{F}_{t}\right], \forall t \geq 0
$$

where the notation $\stackrel{\text { TP }}{=}$ means that the equality follows by the tower property of conditional expectations. From (4.1), we obtain

$$
Y_{t}^{0}=\frac{1}{G_{t}} \int_{t}^{\infty} Y_{t}(u) p_{t}(u) g(u) d u, \forall t \geq 0 .
$$

A particular case occurs when $Y^{\mathbb{G}}$ is the optional projection of a process $Y(\tau)$. In that case, for each $u \geq 0$, one has

$$
Y_{t}^{0}=\frac{1}{G_{t}} \int_{t}^{\infty} Y_{t}(u) p_{t}(u) g(u) d u, \forall t \geq 0, \quad \text { and } \quad Y_{t}^{1}(u)=Y_{t}(u), \forall t \geq u
$$

where the process $G$ is defined in (3.4).

Furthermore, if the process $Y^{\mathbb{G}}$ is $\mathbb{G}_{\text {-predictable }}^{3}$, Lemma 4.4 in [20] or Proposition 2.8, part (ii) in [6] yield the representation

$$
Y_{t}^{\mathbb{G}}=\mathbb{1}_{\{\tau \geq t\}} Y_{t}^{0}+\mathbb{1}_{\{\tau<t\}} Y_{t}^{1}(\tau), \forall t \geq 0,
$$

where the process $Y^{0}$ is $\mathbb{F}$-predictable and $(\omega, t, u) \mapsto Y_{t}^{1}(\omega, u)$ is $\mathcal{P}(\mathbb{F}) \otimes \mathcal{B}\left(\mathbb{R}^{+}\right)$-measurable (we shall say that $Y^{1}$ is a $\mathcal{P}(\mathbb{F}) \otimes \mathcal{B}\left(\mathbb{R}^{+}\right)$-measurable process), and $Y^{0}$ is called the $\mathbb{F}$-predictable reduction of $Y^{\mathbb{G}}$. Note that the process $\left(\mathbb{1}_{\{\tau<t\}}\right)_{t \geq 0}$ is predictable.

Remark 4.5. In our setting, $\mathbb{F}$ being a Brownian filtration, the $\mathbb{F}$-predictable reduction of $Y^{\mathbb{G}}$ and the $\mathbb{F}$-optional reduction are equal. Note that, in particular, the process $Y^{0}$ given by (4.6) is continuous. Obviously, for each $u \geq 0, Y_{t}^{1}(u)=Y_{t}(u)$, for all $t \geq u$. The main difference between (4.5) and (4.7) is the use of the sets $\{\tau \leq t\}$ instead of $\{\tau<t\}$, for all $t \geq 0$.

\footnotetext{
${ }^{3}$ In the literature, the result for predictable processes is known from the seminal work of Jeulin, in a general setting of progressive enlargement by a random time. The result for optional processes is recent, and valid under restrictive conditions such as Jacod's equivalence hypothesis. In our paper, we do need the optional version, because the $\mathbb{G}$-martingales are not all predictable.
} 
Projections of martingales in enlargements of Brownian filtrations

Under Jacod's equivalence hypothesis, the process $M^{\mathbb{G}}=\left(M_{t}^{\mathbb{G}}\right)_{t \geq 0}$ defined by

$$
M_{t}^{\mathbb{G}}=\mathbb{1}_{\{\tau \leq t\}}-\int_{0}^{t} \mathbb{1}_{\{\tau \geq s\}} \frac{p_{s}(s) g(s)}{G_{s}} d s, \forall t \geq 0,
$$

is a $\mathbb{G}$-martingale (see Theorem 2.1 in [15] or Proposition 4.4 in [10]). We denote by $\lambda=\left(\lambda_{t}\right)_{t \geq 0}$ the process

$$
\lambda_{t}=\frac{p_{t}(t) g(t)}{G_{t}}, \forall t \geq 0 .
$$

Then, equation (4.8) can be rewritten as

$$
M_{t}^{\mathrm{G}}=H_{t}^{\mathrm{G}}-\int_{0}^{t}\left(1-H_{s}^{\mathrm{G}}\right) \lambda_{s} d s=H_{t}^{\mathbb{G}}-\int_{0}^{t \wedge \tau} \lambda_{s} d s, \forall t \geq 0,
$$

so that $\lambda$ is the intensity rate of $\tau$. Note that we can apply the result of Chapter II, Theorem 13, page 31 in [5] to prove that it is possible to choose an $\mathbb{F}$-predictable version of the intensity rate and, since the function $g$ is deterministic and $G$ is a continuous $\mathbb{F}$-supermartingale, it is possible to consider in place of $\left(p_{t}(t)\right)_{t \geq 0}$ its $\mathbb{F}$-predictable projection.

Proposition 4.1 in [10] states that

$$
G_{t}=1+M_{t}-\int_{0}^{t} p_{s}(s) g(s) d s, \forall t \geq 0,
$$

where the process $M=\left(M_{t}\right)_{t \geq 0}$ is an $\mathbb{F}$-martingale given by

$$
M_{t}=-\int_{0}^{t}\left(p_{t}(s)-p_{s}(s)\right) g(s) d s, \forall t \geq 0,
$$

and then

$$
G_{t}+\int_{0}^{t} p_{s}(s) g(s) d s=1-\int_{0}^{t}\left(p_{t}(s)-p_{s}(s)\right) g(s) d s, \forall t \geq 0 .
$$

Moreover, we have

$$
p_{t}(s)-p_{s}(s)=\int_{s}^{t} d p_{u}(s)=\int_{s}^{t} p_{u}(s) \varphi_{u}(s) d W_{u}, \forall t \geq s \geq 0,
$$

where the last equality follows by equation (3.3). Replacing the latter equality in the previous one, we get

$$
\begin{aligned}
G_{t}+\int_{0}^{t} p_{s}(s) g(s) d s & =1-\int_{0}^{t}\left(\int_{s}^{t} p_{u}(s) \varphi_{u}(s) d W_{u}\right) g(s) d s \\
& =1-\int_{0}^{t}\left(\int_{0}^{u} p_{u}(s) \varphi_{u}(s) g(s) d s\right) d W_{u}, \forall t \geq 0,
\end{aligned}
$$

so that

$$
d G_{t}+p_{t}(t) g(t) d t=-\left(\int_{0}^{t} p_{t}(s) \varphi_{t}(s) g(s) d s\right) d W_{t}, \quad G_{0}=1 .
$$

Moreover, Proposition 4.7 and Remark 4.8 in [10] state that

$$
\int_{0}^{\infty} p_{t}(s) \varphi_{t}(s) g(s) d s=0, \forall t \geq 0
$$

and therefore, we have

$$
\int_{0}^{t} p_{t}(s) \varphi_{t}(s) g(s) d s=-\int_{t}^{\infty} p_{t}(s) \varphi_{t}(s) g(s) d s, \forall t \geq 0 .
$$


Finally, the previous equality and equation (4.9) imply that the process $G$ admits the representation

$$
d G_{t}=-G_{t} \lambda_{t} d t+\left(\int_{t}^{\infty} p_{t}(s) \varphi_{t}(s) g(s) d s\right) d W_{t}, \quad G_{0}=1 .
$$

The decomposition of the $\mathbb{G}$-special semimartingale $W$ follows from Proposition 4.3 in [6]

$$
W_{t}=W_{t}^{\mathrm{G}}+\int_{0}^{t \wedge \tau} \frac{d\langle W, G\rangle_{s}^{\mathbb{F}}}{G_{s}}+\left.\int_{t \wedge \tau}^{t} \frac{d\langle W, p(u)\rangle_{s}^{\mathbb{F}}}{p_{s}(u)}\right|_{u=\tau}, \forall t \geq 0
$$

where the bracket of the two continuous semimartingales $W$ and $G$ is equal (see Chapter IV, page 128, Definition 1.20 in [24]) to the predictable bracket of $W$ and $G^{c}$, the continuous $\mathbb{F}$-martingale part of $G$. By equation (4.11), we get

$$
G_{t}^{c}=\int_{0}^{t}\left(\int_{u=s}^{\infty} p_{s}(u) \varphi_{s}(u) g(u) d u\right) d W_{s}, \forall t \geq 0 .
$$

Then, it follows from P. Lévy characterisation theorem (see, e.g., Chapter IV, Theorem 3.6, page 150 in [24]) that the process $W^{\mathbb{G}}=\left(W_{t}^{\mathbb{G}}\right)_{t \geq 0}$ defined in (4.12) by

$$
W_{t}^{\mathrm{G}}=W_{t}-\int_{0}^{t} \alpha_{s}^{\mathrm{G}} d s, \forall t \geq 0,
$$

where $\alpha^{\mathbb{G}}$ is the $\mathbb{G}$-predictable process defined by

$$
\alpha_{t}^{\mathrm{G}}=\mathbb{1}_{\{\tau \geq t\}} \frac{1}{G_{t}} \frac{d\left\langle W, G^{c}\right\rangle_{t}^{\mathbb{F}}}{d t}+\mathbb{1}_{\{\tau<t\}} \frac{1}{p_{t}(\tau)} \frac{\left.d\langle W, p(u)\rangle_{t}^{\mathbb{F}}\right|_{u=\tau}}{d t}, \forall t \geq 0,
$$

is a $\mathbb{G}$-standard Brownian motion. Note that the $\mathbb{G}$-predictable process $\alpha^{\mathbb{G}}$ admits the decomposition (4.7), that is,

$$
\alpha_{t}^{\mathrm{G}}=\mathbb{1}_{\{\tau \geq t\}} \alpha_{t}^{0}+\mathbb{1}_{\{\tau<t\}} \alpha_{t}^{1}(\tau), \forall t \geq 0,
$$

where, by virtue of the expression in (4.15) and equality (4.13), the $\mathbb{F}$-predictable processes $\alpha^{0}$ and $\alpha^{1}(u)$, for each $u \geq 0$, are given by

$$
\alpha_{t}^{0}=\frac{1}{G_{t}} \int_{t}^{\infty} \varphi_{t}(u) p_{t}(u) g(u) d u, \forall t \geq 0, \quad \text { and } \quad \alpha_{t}^{1}(u)=\varphi_{t}(u), \forall t \geq u .
$$

Note that, by virtue of equalities (4.2), (4.14) and (4.17), it follows that the $\mathbb{F}^{(\tau)}$ semimartingale representation of the $\mathbb{G}$-martingale $W^{\mathbb{G}}$ turns out to be

$$
W_{t}^{\mathrm{G}}=W_{t}(\tau)-\int_{0}^{t \wedge \tau}\left(\alpha_{s}^{0}-\varphi_{s}(\tau)\right) d s, \forall t \geq 0
$$

where $W(\tau)$ is given by equation (4.2).

From the definition of $\alpha^{0}$ in (4.17) and equality (4.11), we obtain

$$
G_{t}=1-\int_{0}^{t} G_{s} \lambda_{s} d s+\int_{0}^{t} G_{s} \alpha_{s}^{0} d W_{s}, \forall t \geq 0 .
$$

The multiplicative decomposition of the $\mathbb{F}$-supermartingale being $G=N D$, where $N$ is a local martingale and $D$ a decreasing predictable process (see (2.2)), integration by parts leads to

$$
d G_{t}=D_{t} d N_{t}+N_{t} d D_{t}, \quad G_{0}=1 .
$$


Due to the uniqueness of the Doob-Meyer decomposition obtained in (4.19), one has

$$
D_{t} d N_{t}=G_{t} \alpha_{t}^{0} d W_{t} \text { and } N_{t} d D_{t}=-G_{t} \lambda_{t} d t
$$

which is equivalent to

$$
d D_{t}=-D_{t} \lambda_{t} d t \quad \text { and } \quad d N_{t}=N_{t} \alpha_{t}^{0} d W_{t}
$$

Solving the latter stochastic differential equations and applying the initial conditions $G_{0}=1$ and $N_{0}=1$, it follows that $G=N D$ with

$$
\begin{aligned}
& D_{t}=\exp \left(-\int_{0}^{t} \lambda_{s} d s\right), \forall t \geq 0 \\
& N_{t}=\exp \left(\int_{0}^{t} \alpha_{s}^{0} d W_{s}-\frac{1}{2} \int_{0}^{t}\left(\alpha_{s}^{0}\right)^{2} d s\right), \forall t \geq 0 .
\end{aligned}
$$

Finally, we recall that the pair $\left(W^{\mathbb{G}}, M^{\mathbb{G}}\right)$ enjoys the $\mathbb{G}$-predictable representation property, that is, any $\mathbb{G}$-martingale $Y^{\mathbb{G}}$ admits the integral representation

$$
Y_{t}^{\mathbb{G}}=Y_{0}^{\mathbb{G}}+\int_{0}^{t} \beta_{s}^{\mathbb{G}} d W_{s}^{\mathbb{G}}+\int_{0}^{t} \gamma_{s}^{\mathbb{G}} d M_{s}^{\mathbb{G}}, \forall t \geq 0,
$$

with some $\mathbb{G}$-predictable processes $\beta^{\mathbb{G}}$ and $\gamma^{\mathbb{G}}$ (see Proposition 5.5 (ii) in [6] or Theorem 6.4 in [18]).

Remark 4.6. Note that, letting

$$
\gamma_{t}^{\mathbb{G}}=\mathbb{1}_{\{\tau \geq t\}} \gamma_{t}^{0}+\mathbb{1}_{\{\tau<t\}} \gamma_{t}^{1}(\tau), \forall t \geq 0,
$$

instead, the equality

$$
\int_{0}^{t} \gamma_{s}^{\mathbb{G}} d M_{s}^{\mathbb{G}}=\int_{0}^{t} \gamma_{s}^{0} d M_{s}^{\mathbb{G}}, \forall t \geq 0,
$$

holds for any choice of the $\mathcal{P}(\mathbb{F}) \otimes \mathcal{B}\left(\mathbb{R}^{+}\right)$-measurable process $\gamma^{1}$, since $M^{\mathbb{G}}$ is flat after $\tau$ (i.e., $M_{t}^{\mathbb{G}}=M_{t \wedge \tau}^{\mathbb{G}}$, for all $t \geq 0$ ) and equality (4.22) can be simplified to

$$
Y_{t}^{\mathbb{G}}=Y_{0}^{\mathbb{G}}+\int_{0}^{t} \beta_{s}^{\mathbb{G}} d W_{s}^{\mathbb{G}}+\int_{0}^{t} \gamma_{s}^{0} d M_{s}^{\mathbb{G}}, \forall t \geq 0 .
$$

\section{Optional projections of martingales}

Let $Y(\tau)$ be an $\mathbb{F}^{(\tau)}$-martingale. Then, due to the $\mathbb{F}^{(\tau)}$-predictable representation property for $W(\tau)$, the martingale $Y(\tau)$ admits the integral representation given by (4.3). We study the $\mathbb{G}$-optional projection $Y^{\mathbb{G}}$ of the process $Y(\tau)$. By Remark 2.2, it follows that $Y^{\mathbb{G}}$ is a $\mathbb{G}$-martingale. Any $\mathbb{G}$-martingale $Y^{\mathbb{G}}$ admits the integral representation given by (4.22), with some $\mathbb{G}$-predictable processes $\beta^{\mathbb{G}}$ and $\gamma^{\mathbb{G}}$ that can be represented as

$$
\begin{aligned}
& \beta_{t}^{\mathbb{G}}=\mathbb{1}_{\{\tau \geq t\}} \beta_{t}^{0}+\mathbb{1}_{\{\tau<t\}} \beta_{t}^{1}(\tau), \forall t \geq 0, \\
& \gamma_{t}^{\mathbb{G}}=\mathbb{1}_{\{\tau \geq t\}} \gamma_{t}^{0}, \forall t \geq 0,
\end{aligned}
$$

where, as in (4.7), $\beta^{0}, \gamma^{0}$ are $\mathbb{F}$-predictable processes and $\beta^{1}$ is a $\mathcal{P}(\mathbb{F}) \otimes \mathcal{B}\left(\mathbb{R}^{+}\right)$measurable process. We also consider $Y$, the $\mathbb{F}$-optional projection of a $\mathbb{G}$-martingale $Y^{\mathbb{G}}$. By Remark 2.2, $Y$ is an $\mathbb{F}$-martingale. 
Furthermore, due to the $\mathbb{F}$-predictable representation property of $W$, any $\mathbb{F}$-martingale (in particular the $\mathbb{F}$-optional projection of $Y(\tau)$ and the $\mathbb{F}$-optional projection of $Y^{\mathbb{G}}$ ) admits the integral representation

$$
Y_{t}=Y_{0}+\int_{0}^{t} \sigma_{s} d W_{s}, \forall t \geq 0,
$$

where $\sigma$ is a suitable $\mathbb{F}$-predictable process. In the next subsections, we will show how to compute the processes $\beta^{\mathbb{G}}, \gamma^{0}$ in terms of the processes $Y(\tau)$ and $y(\tau)$ and give the expression of $\sigma$ in terms of $Y^{\mathbb{G}}$ and $\beta^{\mathbb{G}}$.

\subsection{The projections of $\mathbb{F}^{(\tau)}$-martingales on $\mathbb{G}$}

Proposition 5.1. Let the process $Y(\tau)$ be an $\mathbb{F}^{(\tau)}$-martingale with the representation (4.3). Then, its $\mathbb{G}$-optional projection $Y^{\mathbb{G}}=\left(Y_{t}^{\mathbb{G}}\right)_{t \geq 0}$ enjoys representation (4.24) with $Y_{0}^{\mathrm{G}}=\mathbb{E}\left[Y_{0}(\tau)\right]$ and $\beta^{\mathrm{G}}$ as in (5.1), where processes $\beta^{0}, \beta^{1}(u), u \geq 0$, and $\gamma^{0}$ from (5.1) and (5.2), respectively, are given by

$$
\begin{aligned}
\beta_{t}^{0} & =\frac{1}{G_{t}} \int_{t}^{\infty}\left(y_{t}(u)+\left(\varphi_{t}(u)-\alpha_{t}^{0}\right) Y_{t}(u)\right) p_{t}(u) g(u) d u, \forall t \geq 0, \\
\beta_{t}^{1}(u) & =y_{t}(u), \forall t \geq 0, \\
\gamma_{t}^{0} & ={ }^{p} Y_{t}(t)-Y_{t}^{0}, \forall t \geq 0,
\end{aligned}
$$

where $\alpha^{0}$ is given by (4.17), $Y^{0}$ is the $\mathbb{F}$-predictable reduction of $Y^{\mathrm{G}}$ given by (4.6), and $\left({ }^{p} Y_{t}(t)\right)_{t \geq 0}$ is the $\mathbb{F}$-predictable projection of $\left(Y_{t}(t)\right)_{t \geq 0}$.

Proof. In the first part of the proof (the first and the second step), we assume that the martingale $Y(\tau)$ is square integrable ${ }^{4}$, so that the martingale $Y^{\mathbb{G}}$ is square integrable too, hence

$$
\mathbb{E}\left[\int_{0}^{\infty}\left(\left(\beta_{s}^{\mathrm{G}}\right)^{2}+\left(\gamma_{s}^{0}\right)^{2}\left(1-H_{s}^{\mathrm{G}}\right) \lambda_{s}\right) d s\right]<\infty .
$$

In the first step, we determine $\beta^{\mathbb{G}}$ and, in the second step, we determine $\gamma^{0}$. We generalize the result to any $\mathbb{F}^{(\tau)}$-martingale by localisation in the second part of the proof (third step).

First step: Let us determine the process $\beta^{\mathbb{G}}$ which, due to the square integrability condition on $Y(\tau)$, satisfies

$$
\mathbb{E}\left[\int_{0}^{\infty}\left(\beta_{s}^{\mathbb{G}}\right)^{2} d s\right]<\infty .
$$

For this purpose, we consider a bounded $\mathbb{G}$-predictable process $n^{\mathbb{G}}$ and define $V^{\mathbb{G}}=$ $\left(V_{t}^{\mathrm{G}}\right)_{t \geq 0}$ by

$$
V_{t}^{\mathrm{G}}=\int_{0}^{t} n_{s}^{\mathrm{G}} d W_{s}^{\mathrm{G}}, \forall t \geq 0 .
$$

Since $W^{\mathbb{G}}$ is a $\mathbb{G}$-standard Brownian motion and the process $n^{\mathbb{G}}$ is bounded, the continuous process $V^{\mathbb{G}}$ is a $\mathbb{G}$-martingale. On the one hand, the $\mathbb{G}$-standard Brownian motion $W^{\mathbb{G}}$ is orthogonal to the pure-jump $\mathbb{G}$-martingale $M^{\mathbb{G}}$, leading to the fact that

$$
\mathbb{E}\left[Y_{t}(\tau) V_{t}^{\mathbb{G}}\right] \stackrel{\mathrm{TP}}{=} \mathbb{E}\left[Y_{t}^{\mathbb{G}} V_{t}^{\mathbb{G}}\right]=\mathbb{E}\left[V_{t}^{\mathbb{G}} \int_{0}^{t} \beta_{s}^{\mathbb{G}} d W_{s}^{\mathbb{G}}\right]=\mathbb{E}\left[\int_{0}^{t} \beta_{s}^{\mathbb{G}} n_{s}^{\mathbb{G}} d s\right], \forall t \geq 0 .
$$

\footnotetext{
${ }^{4} \mathrm{~A}$ martingale $X$ is square integrable when the condition $\sup _{t \geq 0} \mathbb{E}\left[X_{t}^{2}\right]<\infty$ holds (see Chapter I, Definition 1.41, page 10 in [16]).
} 
Projections of martingales in enlargements of Brownian filtrations

On the other hand, by means of integration by parts for semimartingales, we get

$$
Y_{t}(\tau) V_{t}^{\mathrm{G}}=\int_{0}^{t} Y_{s}(\tau) d V_{s}^{\mathrm{G}}+\int_{0}^{t} V_{s}^{\mathrm{G}} d Y_{s}(\tau)+\left[Y(\tau), V^{\mathrm{G}}\right]_{t}, \forall t \geq 0,
$$

where the first integral on the right-hand side of equation (5.8) is understood as the integral of the $\mathbb{F}^{(\tau)}$-predictable process $Y(\tau)$ with respect to the process $V^{\mathbb{G}}$, considered as an $\mathbb{F}^{(\tau)}$-semimartingale (see (5.10) below), and $\left[Y(\tau), V^{\mathbb{G}}\right]$ is the covariation process of the $\mathbb{F}^{(\tau)}$-semimartingales $Y(\tau)$ and $V^{\mathbb{G}}$, and hence,

$$
\mathbb{E}\left[Y_{t}(\tau) V_{t}^{\mathbb{G}}\right]=\mathbb{E}\left[\int_{0}^{t} Y_{s}(\tau) d V_{s}^{\mathbb{G}}\right]+\mathbb{E}\left[\int_{0}^{t} V_{s}^{\mathbb{G}} d Y_{s}(\tau)\right]+\mathbb{E}\left[\left[Y(\tau), V^{\mathbb{G}}\right]_{t}\right], \forall t \geq 0 .
$$

Now, we develop the expressions in the right-hand side of equality (5.9), which consists of three terms.

- As far as the first term is concerned, we note that, by virtue of equality (4.18), the $\mathrm{F}^{(\tau)}$-semimartingale decomposition of the $\mathbb{G}$-martingale $V^{\mathbb{G}}$ is

$$
V_{t}^{\mathbb{G}}=\int_{0}^{t} n_{s}^{\mathbb{G}} d W_{s}(\tau)+\int_{0}^{t} \mathbb{1}_{\{\tau \geq s\}}\left(\varphi_{s}(\tau)-\alpha_{s}^{0}\right) n_{s}^{\mathbb{G}} d s, \forall t \geq 0 .
$$

The process $\bar{Y}(\tau)=\left(\bar{Y}_{t}(\tau)\right)_{t \geq 0}$ defined by

$$
\bar{Y}_{t}(\tau)=\int_{0}^{t} Y_{s}(\tau) n_{s}^{\mathbb{G}} d W_{s}(\tau), \forall t \geq 0,
$$

is an $\mathbb{F}^{(\tau)}$-martingale, due to the fact that $n^{\mathbb{G}}$ is bounded and that, $Y(\tau)$ being square integrable, we have

$$
\mathbb{E}\left[\int_{0}^{t} Y_{s}^{2}(\tau) d s\right] \leq t \sup _{0 \leq s \leq t} \mathbb{E}\left[Y_{s}^{2}(\tau)\right]<\infty, \forall t \geq 0 .
$$

Then, from (5.10), we have

$$
\mathbb{E}\left[\int_{0}^{t} Y_{s}(\tau) d V_{s}^{\mathbb{G}}\right]=\mathbb{E}\left[\int_{0}^{t} \mathbb{1}_{\{\tau \geq s\}} Y_{s}(\tau)\left(\varphi_{s}(\tau)-\alpha_{s}^{0}\right) n_{s}^{\mathbb{G}} d s\right], \forall t \geq 0 .
$$

- In order to handle the second term it is enough to prove that the $\mathbb{F}^{(\tau)}$-local martingale $M(\tau)=\left(M_{t}(\tau)\right)_{t \geq 0}$ defined by

$$
M_{t}(\tau)=\int_{0}^{t} V_{s}^{\mathbb{G}} d Y_{s}(\tau), \forall t \geq 0
$$

is a true martingale. This will be the case when, for any $T>0$ fixed, the property

$$
\mathbb{E}\left[\sup _{0 \leq t \leq T}\left|M_{t}(\tau)\right|\right]<\infty
$$

holds (see Chapter I, Theorem 51, page 38 in [23]). By Burkholder-Davis-Gundy's inequality ${ }^{5}$, this condition is satisfied if

$$
\mathbb{E}\left[\left(\langle M(\tau)\rangle_{T}^{\mathbb{F}^{(\tau)}}\right)^{1 / 2}\right]<\infty .
$$

\footnotetext{
${ }^{5}$ Burkholder-Davis-Gundy's inequality states that, if $M$ is a local martingale, for any $p \geq 1$, then the expression

$$
\mathbb{E}\left[\sup _{0 \leq t \leq T}\left|M_{t}\right|^{p}\right] \leq C_{p} \mathbb{E}\left[\left\langle M_{T}\right\rangle^{p / 2}\right]
$$

holds, for some $C_{p}>0$ depending on $p$ only (see, e.g., Chapter IV, Section 4, Theorem 48, page 195 in [23]).
} 
Note that we have

$$
\begin{aligned}
& \mathbb{E}\left[\left(\langle M(\tau)\rangle_{T}^{\mathbb{F}^{(\tau)}}\right)^{1 / 2}\right]=\mathbb{E}\left[\left(\int_{0}^{T}\left(V_{s}^{\mathbb{G}}\right)^{2} y_{s}^{2}(\tau) d s\right)^{1 / 2}\right] \\
& \leq \mathbb{E}\left[\sup _{0 \leq s \leq T}\left|V_{s}^{\mathbb{G}}\right|\left(\int_{0}^{T} y_{s}^{2}(\tau) d s\right)^{1 / 2}\right] \leq \mathbb{E}\left[\sup _{0 \leq s \leq T}\left|V_{s}^{\mathbb{G}}\right|^{2}\right]+\mathbb{E}\left[\int_{0}^{T} y_{s}^{2}(\tau) d s\right],
\end{aligned}
$$

where we have used the fact that $|a b| \leq\left(a^{2}+b^{2}\right)$, for any $a, b \in \mathbb{R}$. It follows, using again Burkholder-Davis-Gundy's inequality, that

$$
\mathbb{E}\left[\sup _{0 \leq s \leq T}\left|V_{s}^{\mathbb{G}}\right|^{2}\right] \leq C_{2} \mathbb{E}\left[\int_{0}^{T}\left(n_{s}^{\mathbb{G}}\right)^{2} d s\right]<\infty,
$$

for some constant $C_{2}>0$. Moreover, by the assumption of square integrability of the $\mathbb{F}^{(\tau)}$-martingale $Y(\tau)$, we have

$$
\mathbb{E}\left[\int_{0}^{T} y_{s}^{2}(\tau) d s\right]<\infty .
$$

Hence, the second integral on the right-hand side of equation (5.8) is a centered $\mathbb{F}^{(\tau)}$ martingale, so that the second term at the right-hand side of equality (5.9) is identically zero.

- Finally, as far as the third term is concerned, due to the continuity of the process $Y(\tau)$, and, since the continuous $\mathbb{F}^{(\tau)}$-martingale part of the $\mathbb{F}^{(\tau)}$-semimartingale $V^{\mathrm{G}}$ is $\bar{V}^{\mathrm{G}}=\left(\bar{V}_{t}^{\mathrm{G}}\right)_{t \geq 0}$ defined by

$$
\bar{V}_{t}^{\mathrm{G}}=\int_{0}^{t} n_{s}^{\mathrm{G}} d W_{s}(\tau), \forall t \geq 0,
$$

we obtain ${ }^{6}$

$$
\left[Y(\tau), V^{\mathbb{G}}\right]_{t}=\left\langle Y(\tau), \bar{V}^{\mathbb{G}}\right\rangle_{t}^{\mathbb{F}^{(\tau)}}=\int_{0}^{t} y_{s}(\tau) n_{s}^{\mathbb{G}} d s, \forall t \geq 0 .
$$

Summarising, we get

$$
\mathbb{E}\left[Y_{t}(\tau) V_{t}^{\mathbb{G}}\right]=\mathbb{E}\left[\int_{0}^{t}\left(y_{s}(\tau)+\mathbb{1}_{\{\tau \geq s\}}\left(\varphi_{s}(\tau)-\alpha_{s}^{0}\right) Y_{s}(\tau)\right) n_{s}^{\mathbb{G}} d s\right], \forall t \geq 0 .
$$

By applying Fubini's theorem twice to interchange the order of expectation and integration, we obtain, from equalities (5.7) and (5.11), the equality

$$
\begin{aligned}
& \mathbb{E}\left[\int_{0}^{t} \beta_{s}^{\mathrm{G}} n_{s}^{\mathrm{G}} d s\right]=\mathbb{E}\left[Y_{t}(\tau) V_{t}^{\mathrm{G}}\right] \\
& \stackrel{\mathrm{TP}}{=} \mathbb{E}\left[\int_{0}^{t} \mathbb{E}\left[y_{s}(\tau)+\mathbb{1}_{\{\tau \geq s\}}\left(\varphi_{s}(\tau)-\alpha_{s}^{0}\right) Y_{s}(\tau) \mid \mathcal{G}_{s}\right] n_{s}^{\mathrm{G}} d s\right], \forall t \geq 0,
\end{aligned}
$$

for any $\mathbb{G}$-predictable bounded process $n^{\mathbb{G}}$, and, since $\tau$ is a $\mathbb{G}$-stopping time, we have

$$
\begin{aligned}
\beta_{t}^{\mathrm{G}} & =\mathbb{E}\left[y_{t}(\tau)+\mathbb{1}_{\{\tau \geq t\}}\left(\varphi_{t}(\tau)-\alpha_{t}^{0}\right) Y_{t}(\tau) \mid \mathcal{G}_{t}\right] \\
& =\mathbb{1}_{\{\tau \geq t\}} \mathbb{E}\left[y_{t}(\tau)+\left(\varphi_{t}(\tau)-\alpha_{t}^{0}\right) Y_{t}(\tau) \mid \mathcal{G}_{t}\right]+\mathbb{1}_{\{t>\tau\}} y_{t}(\tau), \forall t \geq 0 .
\end{aligned}
$$

\footnotetext{
${ }^{6}$ For any two semimartingales $X=\left(X_{t}\right)_{t \geq 0}$ and $Y=\left(Y_{t}\right)_{t \geq 0}$, one has

$$
[X, Y]_{t}=\left\langle X^{c}, Y^{c}\right\rangle_{t}+\sum_{0 \leq s \leq t} \Delta X_{s} \Delta Y_{s}, \forall t \geq 0
$$

where $\Delta X_{t}=X_{t}-X_{t-}$ and $\Delta Y_{t}=Y_{t}-Y_{t-}$ (see Chapter VIII, Definition 8.2, page 209 in [14]).
} 
Projections of martingales in enlargements of Brownian filtrations

Then, by equality (4.1), applied to

$$
\mathbb{E}\left[y_{t}(\tau)+\left(\varphi_{t}(\tau)-\alpha_{t}^{0}\right) Y_{t}(\tau) \mid \mathcal{G}_{t}\right], \forall t \geq 0,
$$

the $\mathbb{F}$-predictable reduction of $\beta^{\mathbb{G}}$ is given by

$$
\beta_{t}^{0}=\frac{1}{G_{t}} \int_{t}^{\infty}\left(y_{t}(u)+\left(\varphi_{t}(u)-\alpha_{t}^{0}\right) Y_{t}(u)\right) p_{t}(u) g(u) d u, \forall t \geq 0,
$$

and (5.12) leads to $\beta_{t}^{1}(u)=y_{t}(u)$, for all $t \geq u$ and each $u \geq 0$.

Second step: We now determine the process $\gamma^{0}$. On the one hand, for any bounded G-predictable process $n^{\mathbb{G}}$, using the facts that $M^{\mathbb{G}}$ is a $\mathbb{G}$-martingale strongly orthogonal to $W^{\mathrm{G}}$ such that the equality

$$
\left\langle M^{\mathbb{G}}\right\rangle_{t}^{\mathbb{G}}=\int_{0}^{t} \mathbb{1}_{\{\tau>s\}} \lambda_{s} d s
$$

holds, and that $M^{\mathbb{G}}$ is flat after $\tau$, we have

$$
\begin{aligned}
& \mathbb{E}\left[Y_{t}(\tau) \int_{0}^{t} n_{s}^{\mathbb{G}} d M_{s}^{\mathbb{G}}\right]=\mathbb{E}\left[Y_{t}(\tau) \int_{0}^{t} n_{s}^{0} d M_{s}^{\mathbb{G}}\right] \\
& \stackrel{\mathrm{TP}}{=} \mathbb{E}\left[Y_{t}^{\mathbb{G}} \int_{0}^{t} n_{s}^{0} d M_{s}^{\mathbb{G}}\right]=\mathbb{E}\left[\int_{0}^{t} \gamma_{s}^{0} d M_{s}^{\mathbb{G}} \int_{0}^{t} n_{s}^{0} d M_{s}^{\mathbb{G}}\right] \\
& =\mathbb{E}\left[\int_{0}^{t} \mathbb{1}_{\{\tau>s\}} \gamma_{s}^{0} \lambda_{s} n_{s}^{0} d s\right] \stackrel{\mathrm{TP}}{=} \mathbb{E}\left[\int_{0}^{t} \gamma_{s}^{0} \lambda_{s} G_{s} n_{s}^{0} d s\right], \forall t \geq 0,
\end{aligned}
$$

where $\lambda$ is, by abuse of notation, the F-predictable version of the process defined in (4.9), and $n^{0}$ is the $\mathbb{F}$-predictable reduction of $n^{\mathbb{G}}$. We also note that $M^{\mathbb{G}}$ is an $\mathbb{F}^{(\tau)}$-predictable bounded variation process. Applying the integration by parts formula and using the fact that the covariation process of the $\mathbb{F}^{(\tau)}$-martingale $Y(\tau)$ and the $\mathbb{F}^{(\tau)}$-predictable bounded variation semimartingale $U^{\mathbb{G}}=\left(U_{t}^{\mathbb{G}}\right)_{t \geq 0}$ defined by

$$
U_{t}^{\mathrm{G}}=\int_{0}^{t} n_{s}^{0} d M_{s}^{\mathrm{G}}, \forall t \geq 0,
$$

is null, we obtain

$$
\mathbb{E}\left[Y_{t}(\tau) U_{t}^{\mathbb{G}}\right]=\mathbb{E}\left[\int_{0}^{t} Y_{s}(\tau) n_{s}^{0} d M_{s}^{\mathbb{G}}+\int_{0}^{t} U_{s}^{\mathbb{G}} d Y_{s}(\tau)\right], \forall t \geq 0 .
$$

Applying arguments similar to the ones used in the first part of the proof, we obtain that the $\mathbb{F}^{(\tau)}$-local martingale $\bar{U}^{\mathrm{G}}=\left(\bar{U}_{t}^{\mathrm{G}}\right)_{t \geq 0}$ given by

$$
\bar{U}_{t}^{\mathrm{G}}=\int_{0}^{t} U_{s}^{\mathbb{G}} d Y_{s}(\tau), \forall t \geq 0,
$$

is an $\mathbb{F}^{(\tau)}$-martingale.

Moreover, we have

$$
\begin{aligned}
& \mathbb{E}\left[\int_{0}^{t} Y_{s}(\tau) n_{s}^{0} d M_{s}^{\mathrm{G}}\right]=\mathbb{E}\left[\int_{0}^{t} Y_{s}(\tau) n_{s}^{0} d H_{s}^{\mathrm{G}}-\int_{0}^{t} \mathbb{1}_{\{\tau>s\}} \lambda_{s} Y_{s}(\tau) n_{s}^{0} d s\right] \\
& \stackrel{\mathrm{TP}}{=} \mathbb{E}\left[\mathbb{1}_{\{\tau \leq t\}} Y_{\tau}(\tau) n_{\tau}^{0}-\int_{0}^{t} \mathbb{E}\left[Y_{s}(\tau) \mid \mathcal{G}_{s}\right] n_{s}^{0} \lambda_{s} \mathbb{1}_{\{\tau>s\}} d s\right] \\
& \stackrel{\mathrm{TP}}{=} \mathbb{E}\left[\mathbb{1}_{\{\tau \leq t\}} Y_{\tau}(\tau) n_{\tau}^{0}-\int_{0}^{t} Y_{s}^{0} n_{s}^{0} \lambda_{s} G_{s} d s\right], \forall t \geq 0,
\end{aligned}
$$


where $Y^{0}$ is the $\mathbb{F}$-predictable reduction of $Y^{\mathbb{G}}$. Applying equality (4.1) and recalling that, for each $u \geq 0$, the process $Y(u) p(u)$ is an F-martingale (see Proposition 3.1 in [6]), we get

$$
\begin{aligned}
& \mathbb{E}\left[Y_{t}(\tau) n_{\tau}^{0} \mathbb{1}_{\{\tau \leq t\}}\right] \\
& =\mathbb{E}\left[\int_{0}^{t} Y_{t}(u) p_{t}(u) n_{u}^{0} g(u) d u\right] \stackrel{\mathrm{TP}}{=} \mathbb{E}\left[\int_{0}^{t} Y_{u}(u) p_{u}(u) n_{u}^{0} g(u) d u\right], \forall t \geq 0 .
\end{aligned}
$$

We may therefore conclude that, for all $t \geq 0$, for any $\mathbb{F}$-predictable bounded process $n^{0}$, we have

$$
\mathbb{E}\left[\int_{0}^{t} \gamma_{s}^{0} \lambda_{s} G_{s} n_{s}^{0} d s\right]=\mathbb{E}\left[\int_{0}^{t}\left(Y_{s}(s) p_{s}(s) g(s)-\lambda_{s} G_{s} Y_{s}^{0}\right) n_{s}^{0} d s\right], \forall t \geq 0,
$$

and, using the fact that $\lambda_{t} G_{t}=p_{t}(t) g(t)$, for all $t \geq 0$, we get that, for any $\mathbb{F}$-predictable bounded process $n^{0}$,

$$
\mathbb{E}\left[\int_{0}^{t}\left(\gamma_{s}^{0}-Y_{s}(s)+Y_{s}^{0}\right) \lambda_{s} G_{s} n_{s}^{0} d s\right]=0, \forall t \geq 0,
$$

that is, equality (5.6) holds ${ }^{7}$, using tower property, the fact that $G$ is predictable and that one can choose a predictable version of $\lambda$.

Third step: The result obtained for square integrable martingales $Y(\tau)$ can be extended by means of localization to the case of martingales using standard methods. More precisely, if $Y(\tau)$ is an $\mathbb{F}^{(\tau)}$-martingale, one can introduce a localizing sequence $\left(T_{k}\right)_{k \geq 1}$ of $\mathbb{F}^{(\tau)}$-stopping times so that the stopped processes $Y^{T_{k}}(\tau)=\left(Y_{t \wedge T_{k}}(\tau)\right)_{t \geq 0}$, $k \geq 1$, are square integrable martingales. Then, the arguments of the first and the second step of the proof lead to

$$
\begin{aligned}
& \mathbb{E}\left[\int_{0}^{T_{k}} \beta_{s}^{\mathbb{G}} n_{s}^{\mathbb{G}} d s\right] \\
& =\mathbb{E}\left[\int_{0}^{\infty} \mathbb{E}\left[\mathbb{1}_{\left\{s \leq T_{k}\right\}}\left(y_{s}(\tau)+\mathbb{1}_{\{\tau \geq s\}}\left(\varphi_{s}(\tau)-\alpha_{s}^{0}\right) Y_{s}(\tau)\right) \mid \mathcal{G}_{s}\right] n_{s}^{\mathbb{G}} d s\right], \forall t \geq 0 .
\end{aligned}
$$

Hence, taking into account the fact that $T_{k}$ may fail to be a $\mathbb{G}$-stopping time, we obtain

$$
\beta_{t}^{\mathrm{G}} \mathbb{P}\left(t \leq T_{k} \mid \mathcal{G}_{t}\right)=\mathbb{E}\left[\mathbb{1}_{\left\{t \leq T_{k}\right\}}\left(y_{t}(\tau)+\mathbb{1}_{\{\tau \geq t\}}\left(\varphi_{t}(\tau)-\alpha_{t}^{0}\right) Y_{t}(\tau)\right) \mid \mathcal{G}_{t}\right], \forall t \geq 0,
$$

and, letting $k$ going to infinity, since $\mathbb{1}_{\left\{t \leq T_{k}\right\}}$ increases to one, for each $t \geq 0$, we get the result. Similar arguments are applied to obtain $\gamma^{0}$.

Remark 5.2. One cannot extend the result for $\mathbb{F}^{(\tau)}$-local martingales, since if $Y(\tau)$ is an $\mathbb{F}^{(\tau)}$-local martingale, its $\mathbb{G}$-optional projection may fail to be a $\mathbb{G}$-local martingale.

\subsection{The projections of $\mathbb{F}^{(\tau)}$-martingales on $\mathbb{F}$}

Proposition 5.3. Let $Y(\tau)$ be an $\mathbb{F}^{(\tau)}$-martingale with the representation given by equality (4.3). Then, its $\mathbb{F}$-optional projection $Y$ admits the representation (5.3) with the $\mathbb{F}$-predictable process $\sigma$ given by

$$
\sigma_{t}=\int_{0}^{\infty}\left(y_{t}(u)+\varphi_{t}(u) Y_{t}(u)\right) p_{t}(u) g(u) d u, \forall t \geq 0 .
$$

\footnotetext{
${ }^{7}$ We are not able to give conditions such that $\left(Y_{t}(t), t \geq 0\right)$ is predictable.
} 
Proof. It follows from the predictable representation property in the filtration $\mathbb{F}$ that there exists an $\mathbb{F}$-predictable process $\sigma$ such that equality (5.3) holds, for all $t \geq 0$. We assume that $Y(\tau)$ is a square integrable martingale. On the one hand, for any bounded F-predictable process $n=\left(n_{t}\right)_{t \geq 0}$, we have

$$
\mathbb{E}\left[Y_{t}(\tau) \int_{0}^{t} n_{s} d W_{s}\right] \stackrel{\mathrm{TP}}{=} \mathbb{E}\left[Y_{t} \int_{0}^{t} n_{s} d W_{s}\right]=\mathbb{E}\left[\int_{0}^{t} \sigma_{s} n_{s} d s\right], \forall t \geq 0 .
$$

On the other hand, using the representation in (4.2) and applying the integration by parts formula to the left-hand side of (5.14), we get

$$
\begin{aligned}
& \mathbb{E}\left[Y_{t}(\tau) \int_{0}^{t} n_{s} d W_{s}\right]=\mathbb{E}\left[Y_{t}(\tau)\left(\int_{0}^{t} n_{s} d W_{s}(\tau)+\int_{0}^{t} \varphi_{s}(\tau) n_{s} d s\right)\right] \\
& =\mathbb{E}\left[\int_{0}^{t}\left(y_{s}(\tau)+\varphi_{s}(\tau) Y_{s}(\tau)\right) n_{s} d s\right] \stackrel{\text { TP }}{=} \mathbb{E}\left[\int_{0}^{t} \mathbb{E}\left[y_{s}(\tau)+\varphi_{s}(\tau) Y_{s}(\tau) \mid \mathcal{F}_{s}\right] n_{s} d s\right], \forall t \geq 0 .
\end{aligned}
$$

Hence, by equation (4.1), we have

$$
\sigma_{t}=\mathbb{E}\left[y_{t}(\tau)+\varphi_{t}(\tau) Y_{t}(\tau) \mid \mathcal{F}_{t}\right]=\int_{0}^{\infty}\left(y_{t}(u)+\varphi_{t}(u) Y_{t}(u)\right) p_{t}(u) g(u) d u, \forall t \geq 0,
$$

that completes the proof in the case of square integrable $\mathbb{F}^{(\tau)}$-martingales. For each $u \geq 0$, the processes $y(u)$ and $\varphi(u)$ being $\mathbb{F}$-predictable, and $Y(u), p(u)$ being $\mathbb{F}$-adapted and continuous, the process $\sigma$ is $\mathbb{F}$-predictable.

The extension of this result to any $\mathbb{F}^{(\tau)}$-martingale is done as in Proposition 5.1.

\subsection{The projections of $\mathbb{G}$-martingales on $\mathbb{F}$}

Proposition 5.4. Let $Y^{\mathbb{G}}=\left(Y_{t}^{\mathbb{G}}\right)_{t \geq 0}$ be a $\mathbb{G}$-martingale with the representation given by equality (4.22). Then, its $\mathbb{F}$-optional projection $Y$ admits the representation

$$
Y_{t}=Y_{0}+\int_{0}^{t} \eta_{s} d W_{s}, \forall t \geq 0,
$$

where the $\mathrm{F}$-predictable process $\eta$ is given by

$$
\eta_{t}=\mathbb{E}\left[\beta_{t}^{\mathbb{G}}+\alpha_{t}^{\mathbb{G}} Y_{t}^{\mathbb{G}} \mid \mathcal{F}_{t}\right], \forall t \geq 0 .
$$

In the particular case, where $Y^{\mathbb{G}}$ is the $\mathbb{G}$-optional projection of an $\mathbb{F}^{(\tau)}$-martingale $Y(\tau)$ with the representation (4.3), one has

$$
\eta_{t}=\left(\beta_{t}^{0}+\alpha_{t}^{0} Y_{t}^{0}\right) G_{t}+\int_{0}^{t}\left(\beta_{t}^{1}(u)+\alpha_{t}^{1}(u) Y_{t}^{1}(u)\right) p_{t}(u) g(u) d u, \forall t \geq 0,
$$

with the supermartingale $G$ given by equality (3.4), the processes $\alpha^{0}, \alpha^{1}(u), u \geq 0$, given by (4.17), the processes $\beta^{0}, \beta^{1}(u), u \geq 0$, given by equality (5.1), and the processes $Y^{0}$, $Y^{1}(u), u \geq 0$, defined by equality (4.6).

Proof. Let $Y^{\mathrm{G}}$ be a $\mathbb{G}$-square integrable martingale, and $n$ be a bounded F-predictable process. On the one hand, we get

$$
\mathbb{E}\left[Y_{t}^{\mathrm{G}} \int_{0}^{t} n_{s} d W_{s}\right] \stackrel{\mathrm{TP}}{=} \mathbb{E}\left[Y_{t} \int_{0}^{t} n_{s} d W_{s}\right]=\mathbb{E}\left[\int_{0}^{t} \eta_{s} n_{s} d s\right], \forall t \geq 0 .
$$

On the other hand, by applying the integration by parts formula, we obtain

$$
\begin{aligned}
& \mathbb{E}\left[Y_{t}^{\mathbb{G}} \int_{0}^{t} n_{s} d W_{s}\right]=\mathbb{E}\left[\int_{0}^{t}\left(\int_{0}^{s} n_{u} d W_{u}\right) d Y_{s}^{\mathbb{G}}+\int_{0}^{t} Y_{s}^{\mathbb{G}} n_{s} d W_{s}+\int_{0}^{t} \beta_{s}^{\mathbb{G}} n_{s} d s\right] \\
& =\mathbb{E}\left[\int_{0}^{t}\left(\beta_{s}^{\mathbb{G}}+\alpha_{s}^{\mathbb{G}} Y_{s}^{\mathbb{G}}\right) n_{s} d s\right] \stackrel{\text { TP }}{=} \mathbb{E}\left[\int_{0}^{t} \mathbb{E}\left[\beta_{s}^{\mathbb{G}}+\alpha_{s}^{\mathbb{G}} Y_{s}^{\mathbb{G}} \mid \mathcal{F}_{s}\right] n_{s} d s\right], \forall t \geq 0,
\end{aligned}
$$


where, in order to handle the stochastic integral with respect to $W$, we use its $\mathbb{G}$ semimartingale decomposition given by equality (4.14). We do stress that the true martingale property of the local martingale terms is proved by using similar arguments as in the proof of Proposition 3.2. Hence, we deduce that, for any bounded F-predictable process $n$, we have

$$
\mathbb{E}\left[\int_{0}^{t} \eta_{s} n_{s} d s\right]=\mathbb{E}\left[\int_{0}^{t} \mathbb{E}\left[\beta_{s}^{\mathbb{G}}+\alpha_{s}^{\mathbb{G}} Y_{s}^{\mathbb{G}} \mid \mathcal{F}_{s}\right] n_{s} d s\right], \forall t \geq 0,
$$

and thus,

$$
\eta_{t}=\mathbb{E}\left[\beta_{t}^{\mathbb{G}}+\alpha_{t}^{\mathbb{G}} Y_{t}^{\mathbb{G}} \mid \mathcal{F}_{t}\right], \forall t \geq 0 .
$$

The predictability of $\eta$ is due to the fact that $\eta$ is $\mathbb{F}$-optional, hence $\mathbb{F}$-predictable. In the particular case where $Y^{\mathbb{G}}$ is the $\mathbb{G}$-optional projection of an $\mathbb{F}^{(\tau)}$-martingale $Y(\tau)$ of the form (4.3),

$$
\eta_{t}=\left(\beta_{t}^{0}+\alpha_{t}^{0} Y_{t}^{0}\right) \mathbb{E}\left[\mathbb{1}_{\{t<\tau\}} \mid \mathcal{F}_{t}\right]+\mathbb{E}\left[\mathbb{1}_{\{\tau \leq t\}}\left(\beta_{t}^{1}(\tau)+\alpha_{t}^{1}(\tau)\right) Y_{t}^{1}(\tau) \mid \mathcal{F}_{t}\right], \forall t \geq 0,
$$

that completes the proof. Note that, recalling equality (4.1), in order to deal correctly with the sets $\{\tau<t\}$ and $\{\tau \leq t\}$, for each $t \geq 0$, we have used Remark 4.5.

The extension of this result to any $\mathbb{G}$-martingale is done as in Proposition 5.1.

Remark 5.5. Let $Y(\tau)$ be an $\mathbb{F}^{(\tau)}$-martingale. Note that, since the following equality holds

$$
\mathbb{E}\left[Y_{t}(\tau) \mid \mathcal{F}_{t}\right] \stackrel{\mathrm{TP}}{=} \mathbb{E}\left[\mathbb{E}\left[Y_{t}(\tau) \mid \mathcal{G}_{t}\right] \mid \mathcal{F}_{t}\right], \forall t \geq 0,
$$

holds, we have $\sigma=\eta$. This is not straightforward to conclude that this equality holds true from the explicit forms given in (5.13) and (5.16). As a check, from (5.12) and the fact that (4.17) shows that $\alpha^{1}(u)=\varphi(u)$, for each $u \geq 0$, we see that

$$
\begin{aligned}
\eta_{t} & =\mathbb{E}\left[\beta_{t}^{\mathbb{G}}+\alpha_{t}^{\mathrm{G}} Y_{t}^{\mathrm{G}} \mid \mathcal{F}_{t}\right] \\
& =\mathbb{E}\left[y_{t}(\tau)+\mathbb{1}_{\{\tau \geq t\}}\left(\varphi_{t}(\tau)-\alpha_{t}^{0}\right) Y_{t}(\tau)+\mathbb{1}_{\{\tau \geq t\}} \alpha_{t}^{0} Y_{t}^{0}+\mathbb{1}_{\{\tau<t\}} \alpha_{t}^{1}(\tau) Y_{t}^{1}(\tau) \mid \mathcal{F}_{t}\right] \\
& =\mathbb{E}\left[y_{t}(\tau)+\varphi_{t}(\tau) Y_{t}(\tau) \mid \mathcal{F}_{t}\right]=\sigma_{t}, \forall t \geq 0,
\end{aligned}
$$

where the last equality comes from the fact that $\mathbb{1}_{\{\tau<t\}} Y_{t}^{1}(\tau)=\mathbb{1}_{\{\tau<t\}} Y_{t}(\tau)$, for each $t \geq 0$ (see equality (4.6)).

\section{Changes of probability measures and applications}

In this section, as an example of application of the results from the previous section, we consider the relationships between strictly positive $\mathbb{F}^{(\tau)}$-martingales (or $\mathbb{G}$ martingales) and their optional projections. Note that, for strictly positive martingales, a direct proof of Proposition 6.1 (based on equivalent changes of probability measures) was given in [27]. We apply the results in a financial market framework to study the set of equivalent martingale measures in different filtrations.

\subsection{Exponential martingales and their projections}

\subsubsection{The projections of strictly positive $\mathbb{F}^{(\tau)}$-martingales on $\mathbb{G}$}

Let $L(\tau)$ be a strictly positive $\mathbb{F}^{(\tau)}$-martingale. Then, in particular, we have $L_{0}(\tau)>0$ $\left(\mathbb{P}\right.$-a.s.), so that we can write $L_{t}(\tau)=L_{0}(\tau) X_{t}(\tau)$, where $X(\tau)$ is a strictly positive $\mathbb{F}^{(\tau)}$ martingale, satisfying the hypotheses of Proposition 2.4 with $M=W(\tau)$. Hence, there 
exists an $\mathbb{F}^{(\tau)}$-predictable process $\zeta(\tau)$ such that $X(\tau)=\mathcal{E}(\zeta(\tau) \cdot W(\tau))$ and the following representation holds

$$
\begin{aligned}
L_{t}(\tau) & =L_{0}(\tau) \mathcal{E}(\zeta(\tau) \cdot W(\tau))_{t} \\
& =L_{0}(\tau) \exp \left(\int_{0}^{t} \zeta_{s}(\tau) d W_{s}(\tau)-\frac{1}{2} \int_{0}^{t} \zeta_{s}^{2}(\tau) d s\right), \forall t \geq 0 .
\end{aligned}
$$

Note that, if $\mathbb{E}\left[L_{0}(\tau)\right]=1$, then we can associate to the strictly positive $\mathbb{F}^{(\tau)}$-martingale $L(\tau)$ the probability measure $\widetilde{\mathbb{P}}$ locally equivalent to $\mathbb{P}$ on the filtration $\mathbb{F}^{(\tau)}$ defined by

$$
\left.\frac{d \widetilde{\mathbb{P}}}{d \mathbb{P}}\right|_{\mathcal{F}_{t}^{(\tau)}}=L_{t}(\tau), \forall t \geq 0
$$

Remark 6.1. The particular choice of $L_{0}(\tau)=1$ (P-a.s.) is equivalent to the property $\underset{\mathbb{P}}{\widetilde{P}}(\tau>u)=\mathbb{P}(\tau>u)$, for each $u \geq 0$. Indeed, since $\tau$ is $\mathcal{F}_{0}^{(\tau)}$-measurable, we have $\widetilde{\mathbb{P}}(\tau>u)=\mathbb{E}\left[L_{0}(\tau) \mathbb{1}_{\{\tau>u\}}\right]$, for each $u \geq 0$. Hence, if $L_{0}(\tau)=1$ (P-a.s.) holds, then we have $\widetilde{\mathbb{P}}(\tau>u)=\mathbb{P}(\tau>u)$, for each $u \geq 0$. Conversely, the equality $\widetilde{\mathbb{P}}(\tau>u)=\mathbb{P}(\tau>u)$, for each $u \geq 0$, implies that $\mathbb{E}\left[L_{0}(\tau) \mathbb{1}_{\{\tau>u\}}\right]=\mathbb{E}\left[\mathbb{1}_{\{\tau>u\}}\right]$, for all $u \geq 0$. Thus, using the fact that the events $\{\tau>u\}, u \geq 0$, generate $\mathcal{F}_{0}^{(\tau)}$, we conclude that $L_{0}(\tau)=1$ (P-a.s.).

We now consider the G-optional projection $L^{\mathbb{G}}=\left(L_{t}^{\mathbb{G}}\right)_{t \geq 0}$ of the strictly positive martingale $L(\tau)$. The same arguments which were used to get equation (6.1) are applied here to prove that $L^{\mathbb{G}}=\mathbb{E}\left[L_{0}(\tau)\right] \mathcal{E}\left(\theta^{\mathbb{G}} \cdot X^{\mathbb{G}}\right)$, where, by the $\mathbb{G}$-predictable representation property of the pair $\left(W^{\mathbb{G}}, M^{\mathbb{G}}\right)$, the $\mathbb{G}$-martingale $\theta^{\mathbb{G}} \cdot X^{\mathbb{G}}$ can be represented as

$$
\left(\theta^{\mathbb{G}} \cdot X^{\mathbb{G}}\right)_{t}=\int_{0}^{t} \mu_{s}^{\mathbb{G}} d W_{s}^{\mathbb{G}}+\int_{0}^{t} \psi_{s}^{0} d M_{s}^{\mathbb{G}}, \forall t \geq 0,
$$

with a $\mathbb{G}$-predictable process $\mu^{\mathbb{G}}$ and an $\mathbb{F}$-predictable process $\psi^{0}$ to be determined (this will be done explicitly in Proposition 6.2). Since $W^{\mathbb{G}}$ and $M^{\mathbb{G}}$ are strongly orthogonal G-martingales, Theorem 38, page 86 in [23] is applied to get

$$
L_{t}^{\mathbb{G}}=\mathbb{E}\left[L_{0}(\tau)\right] \mathcal{E}\left(\mu^{\mathbb{G}} \cdot W^{\mathbb{G}}\right)_{t} \mathcal{E}\left(\psi^{0} \cdot M^{\mathbb{G}}\right)_{t}, \forall t \geq 0 .
$$

Moreover, from the definition of the stochastic exponential in (2.3), and the fact that the continuous martingale part of $M^{\mathrm{G}}$ is null, we have

$$
\mathcal{E}\left(\psi^{0} \cdot M^{\mathbb{G}}\right)_{t}=\left(1+\psi_{\tau}^{0}\right)^{H_{t}^{\mathrm{G}}} \exp \left(-\int_{0}^{t \wedge \tau} \psi_{s}^{0} \lambda_{s} d s\right), \forall t \geq 0,
$$

where we recall that $H_{t}^{\mathbb{G}}=\mathbb{1}_{\{\tau \leq t\}}$, for all $t \geq 0$. The strict positivity of the processes $L^{\mathbb{G}}$ and $\mathcal{E}\left(\mu^{\mathbb{G}} \cdot W^{\mathbb{G}}\right)$ implies the strict positivity of the process $\mathcal{E}\left(\psi^{0} \cdot M^{\mathbb{G}}\right)$, and thus, the property $\psi_{\tau}^{0}>-1$.

Proposition 6.2. Let $L(\tau)=\left(L_{t}(\tau)\right)_{t \geq 0}$ be a strictly positive martingale of the form (6.1). Then, its $\mathbb{G}$-optional projection $L^{\mathbb{G}}$ satisfies (6.3) with the $\mathbb{G}$-predictable processes $\mu^{\mathbb{G}}$ and the $\mathbb{F}$-predictable process $\psi^{0}$ given by

$$
\begin{aligned}
\mu_{t}^{\mathbb{G}}= & \mathbb{1}_{\{\tau \geq t\}} \frac{1}{L_{t}^{0} G_{t}} \int_{t}^{\infty} L_{t}(u)\left(\zeta_{t}(u)+\varphi_{t}(u)-\alpha_{t}^{0}\right) p_{t}(u) g(u) d u \\
& +\mathbb{1}_{\{\tau<t\}} \zeta_{t}(\tau), \forall t \geq 0, \\
\psi_{t}^{0}= & \frac{p\left(L_{t}(t)\right)}{L_{t}^{0}}-1, \forall t \geq 0,
\end{aligned}
$$

where $L^{0}=\left(L_{t}^{0}\right)_{t \geq 0}$ is the $\mathbb{F}$-predictable reduction of $L^{\mathbb{G}}$ defined in (4.6). 
Proof. Consider the $\mathbb{F}^{(\tau)}$-martingale $L(\tau)$ given by equality (6.1). Then it is the unique solution of the stochastic differential equation

$$
d L_{t}(\tau)=L_{t}(\tau) \zeta_{t}(\tau) d W_{t}(\tau), \quad L_{0}(\tau)=\ell(\tau),
$$

where $\ell$ is a given strictly positive Borel function. Moreover, the $\mathbb{G}$-optional projection $L^{\mathbb{G}}$ of $L(\tau)$ satisfies the stochastic differential equation

$$
d L_{t}^{\mathbb{G}}=L_{t-}^{\mathbb{G}}\left(\mu_{t}^{\mathbb{G}} d W_{t}^{\mathbb{G}}+\psi_{t}^{0} d M_{t}^{\mathbb{G}}\right), \quad L_{0}^{\mathbb{G}}=\mathbb{E}\left[L_{0}(\tau)\right] .
$$

Then, Proposition 5.1 applies with $Y(\tau)=L(\tau)$ and $y(u)=L(u) \zeta(u)$, for all $u \geq 0$, and therefore, equalities $\mu_{t}^{\mathbb{G}} L_{t}^{\mathbb{G}}=\beta_{t}^{\mathbb{G}}$ and $\psi_{t}^{0} L_{t-}^{0}=\gamma_{t}^{0}$ hold, for all $t \geq 0$.

Example 6.3. Assume that the $\mathbb{F}^{(\tau)}$-martingale $L(\tau)$ is given by

$$
L_{t}(\tau)=\ell(\tau) \mathcal{E}(-\varphi(\tau) \cdot W(\tau))_{t}=\frac{1}{p_{t}(\tau)}, \forall t \geq 0
$$

where $\ell$ is a given strictly positive Borel function and the process $p(u)$ is given by (3.2), for each $u \geq 0$. Note that the second equality is an easy consequence of (3.3). Indeed, Itô's formula and equation (4.2) lead to

$$
d\left(\frac{1}{p_{t}(\tau)}\right)=\frac{1}{p_{t}(\tau)}\left(\varphi_{t}(\tau) d t+d W_{t}\right)=\frac{1}{p_{t}(\tau)} d W_{t}(\tau), \quad \frac{1}{p_{0}(\tau)}=\ell(\tau) .
$$

In this case, its $\mathbb{G}$-optional projection $L^{\mathbb{G}}=\left(L_{t}^{\mathbb{G}}\right)_{t \geq 0}$ is given by

$$
L_{t}^{\mathbb{G}}=\mathcal{E}\left(\mu^{\mathbb{G}} \cdot W^{\mathbb{G}}\right)_{t} \mathcal{E}\left(\psi^{0} \cdot M^{\mathbb{G}}\right)_{t}, \forall t \geq 0,
$$

with

$$
\mu_{t}^{G}=-\mathbb{1}_{\{\tau \geq t\}} \alpha_{t}^{0}-\mathbb{1}_{\{\tau<t\}} \varphi_{t}(\tau) \quad \text { and } \quad \psi_{t}^{0}=\frac{G_{t}}{p_{t}(t)(1-F(t))}-1, \forall t \geq 0,
$$

where we set $F(t)=\mathbb{P}(\tau \leq t)$, for all $t \geq 0$. Observe that the probability measure defined through (6.2) with this choice of $L(\tau)$ (which is a strictly positive martingale with expectation being equal to one) is a preserving and decoupling measure (see [2] and [13] for a discussion of an important role of this strictly positive $\mathbb{F}^{(\tau)}$-martingale $L(\tau)$ ).

\subsubsection{The projections of strictly positive $\mathbb{F}^{(\tau)}$-martingales on $\mathbb{F}$}

Let $L(\tau)$ be a strictly positive $\mathbb{F}^{(\tau)}$-martingale of the form (6.1). Then, its $\mathbb{F}$-optional projection admits the integral representation

$$
L_{t}=\mathbb{E}\left[L_{0}(\tau)\right]+\int_{0}^{t} L_{s} \xi_{s} d W_{s}, \forall t \geq 0,
$$

where the $\mathbb{F}$-predictable process $\xi=\left(\xi_{t}\right)_{t \geq 0}$ can be derived by applying Proposition 5.3 with $Y(\tau)=L(\tau)$ (so that $Y=L$ and $L \xi=\sigma$ ),

$$
\xi_{t}=\frac{1}{L_{t}} \int_{0}^{\infty} L_{t}(u)\left(\zeta_{t}(u)+\varphi_{t}(u)\right) p_{t}(u) g(u) d u, \forall t \geq 0 .
$$

\subsubsection{The projections of strictly positive $\mathbb{G}$-martingales on $\mathbb{F}$}

From (6.3), any strictly positive $\mathbb{G}$-martingale $L^{\mathrm{G}}=\left(L_{t}^{\mathrm{G}}\right)_{t \geq 0}$ admits the equivalent representation

$$
L_{t}^{\mathbb{G}}=L_{0}^{\mathbb{G}}+\int_{0}^{t} L_{s}^{\mathbb{G}} \mu_{s}^{\mathbb{G}} d W_{s}^{\mathbb{G}}+\int_{0}^{t} L_{s-}^{\mathbb{G}} \psi_{s}^{0} d M_{s}^{\mathbb{G}}, \forall t \geq 0,
$$


and, being a $\mathbb{G}$-optional process, it admits the decomposition

$$
L_{t}^{\mathrm{G}}=\mathbb{1}_{\{\tau>t\}} L_{t}^{0}+\mathbb{1}_{\{\tau \leq t\}} L_{t}^{1}(\tau), \forall t \geq 0,
$$

where the process $L^{0}$ is $\mathbb{F}$-optional and the process $L^{1}$ is $\mathcal{O}(\mathbb{F}) \otimes \mathcal{B}\left(\mathbb{R}^{+}\right)$-measurable. By similar arguments, it follows that its $\mathbb{F}$-optional projection $L=\left(L_{t}\right)_{t \geq 0}$ admits the integral representation

$$
L_{t}=L_{0}^{\mathrm{G}}+\int_{0}^{t} L_{s} \varkappa_{s} d W_{s}, \forall t \geq 0,
$$

where $\varkappa=\left(\varkappa_{t}\right)_{t \geq 0}$ is an $\mathbb{F}$-predictable process. In order to derive $\varkappa$, it suffices to apply Proposition 5.4 with $Y^{\mathbb{G}}=L^{\mathbb{G}}, \beta^{0}=(L \mu)^{0}, \beta^{1}=L^{1} \mu^{1}$ and $\eta=L \varkappa$, so that $Y^{0}=L^{0}$ and $Y=L$. The equality $(L \mu)^{0}=L^{0} \mu^{0}$ follows from the definition of predictable reduction. Therefore, we conclude that

$$
\varkappa_{t}=\frac{1}{L_{t}}\left(L_{t}^{0}\left(\mu_{t}^{0}+\alpha_{t}^{0}\right) G_{t}+\int_{0}^{t} L_{t}^{1}(u)\left(\mu_{t}^{1}(u)+\alpha_{t}^{1}(u)\right) p_{t}(u) g(u) d u\right), \forall t \geq 0 .
$$

\subsection{The equivalent martingale measures}

Let us now consider a model of a financial market in which the risky asset price process $S=\left(S_{t}\right)_{t \geq 0}$ follows the stochastic differential equations

$$
\begin{aligned}
d S_{t}=S_{t}\left(\nu d t+\rho d W_{t}\right) & =S_{t}\left(\left(\nu+\rho \varphi_{t}(\tau)\right) d t+\rho d W_{t}(\tau)\right) \\
& =S_{t}\left(\left(\nu+\rho \alpha_{t}^{\mathrm{G}}\right) d t+\rho d W_{t}^{\mathrm{G}}\right), \quad S_{0}=1,
\end{aligned}
$$

according to the filtrations $\mathbb{F}, \mathbb{F}^{(\tau)}$, and $\mathbb{G}$, respectively, where $\nu$ and $\rho>0$ are some constants. We assume that the riskless asset has a zero interest rate.

It is straightforward to show that, for any strictly positive $\mathcal{B}\left(\mathbb{R}^{+}\right)$-measurable function $u \mapsto L_{0}(u)$ satisfying $\mathbb{E}\left[L_{0}(\tau)\right]=1$, the positive $\mathbb{F}^{(\tau)}$-martingale defined in (6.1) provides the Radon-Nikodym density of an equivalent martingale measure on $\mathbb{F}^{(\tau)}$ for $S$ if and only if

$$
\zeta_{t}(\tau)=-\left(\varphi_{t}(\tau)+\frac{\nu}{\rho}\right), \forall t \geq 0 .
$$

In other terms, the set of $\mathrm{F}^{(\tau)}$-equivalent martingale measures for $S$ is the set of probability measures $\mathbb{P}^{*}$ which are locally equivalent to $\mathbb{P}$ on $\mathbb{F}^{(\tau)}$ with the Radon-Nikodym densities

$$
\left.\frac{d \mathbb{P}^{*}}{d \mathbb{P}}\right|_{\mathcal{F}_{t}^{(\tau)}}=L_{t}^{*}(\tau), \forall t \geq 0,
$$

where $L^{*}(\tau)=\left(L_{t}^{*}(\tau)\right)_{t \geq 0}$ is defined by

$$
L_{t}^{*}(\tau)=L_{0}^{*}(\tau) \mathcal{E}\left(-\left(\varphi(\tau)+\frac{\nu}{\rho}\right) \cdot W(\tau)\right)_{t}, \forall t \geq 0,
$$

and $u \mapsto L_{0}^{*}(u)$ is a strictly positive measurable function satisfying $\mathbb{E}\left[L_{0}^{*}(\tau)\right]=1$. In this model, there exists infinitely many such probability measures, which differ from each other by the choice of the initial value $L_{0}^{*}(\tau)$, that is, by the choice of the law of $\tau$ (under $\left.\mathbb{P}^{*}\right)$, namely,

$$
\mathbb{P}^{*}(\tau>u)=\mathbb{E}\left[L_{0}^{*}(\tau) \mathbb{1}_{\{\tau>u\}}\right]=\int_{u}^{\infty} L_{0}^{*}(v) g(v) d v, \forall u \geq 0 .
$$

Note that, by virtue of Girsanov's theorem, the process $\widehat{W}(\tau)=\left(\widehat{W}_{t}(\tau)\right)_{t \geq 0}$ defined as

$$
\widehat{W}_{t}(\tau)=W_{t}(\tau)+\int_{0}^{t}\left(\varphi_{s}(\tau)+\frac{\nu}{\rho}\right) d s, \forall t \geq 0,
$$


is a $\left(\mathbb{P}^{*}, \mathbb{F}^{(\tau)}\right)$-standard Brownian motion.

Let $\mathcal{P}^{*}$ be the set of $\mathbb{G}$-optional projections $L^{*, \mathbb{G}}$ of $L^{*}(\tau)$, which satisfies (6.3), where the processes $\mu^{\mathbb{G}}$ and $\psi^{0}$ are given by equalities (6.4) and (6.5). More precisely, one has

$$
\begin{aligned}
\mu_{t}^{\mathbb{G}} & =-\mathbb{1}_{\{t \leq \tau\}} \frac{1}{L_{t}^{*, 0} G_{t}} \int_{0}^{t} L_{t}^{*}(u)\left(\frac{\nu}{\rho}+\alpha_{t}^{0}\right) p_{t}(u) g(u) d u-\mathbb{1}_{\{\tau<t\}}\left(\varphi_{t}(\tau)+\frac{\nu}{\rho}\right) \\
& =-\mathbb{1}_{\{\tau \geq t\}}\left(\frac{\nu}{\rho}+\alpha_{t}^{0}\right)-\mathbb{1}_{\{\tau<t\}}\left(\varphi_{t}(\tau)+\frac{\nu}{\rho}\right), \forall t \geq 0 \\
\psi_{t}^{0} & =\frac{L_{t}^{*}(t)}{L_{t}^{*, 0}}-1, \forall t \geq 0
\end{aligned}
$$

where $L^{*, 0}$ is the $\mathbb{F}$-predictable reduction of $L^{*, \mathbb{G}}$. Here, each element of $\mathcal{P}^{*}$ is a (locally) equivalent martingale measure on $\mathbb{G}$. Note that $\mu^{\mathrm{G}}$ does not depend on the choice of $L_{0}^{*}$ (see (6.7)), whereas $\psi^{0}$ depends on it.

The set $\mathcal{P}(\mathbb{G})$ of (locally) equivalent martingale measures on $\mathbb{G}$ corresponds to the set of Radon-Nikodym density processes of the form $\mathcal{E}\left(\mu^{\mathbb{G}} \cdot W^{\mathbb{G}}\right) \mathcal{E}\left(\gamma^{0} \cdot M^{\mathbb{G}}\right)$, where $\mu^{\mathbb{G}}$ is given by equality (6.7), for any F-predictable process $\gamma^{0}=\left(\gamma_{t}^{0}\right)_{t \geq 0}$ such that $\gamma_{t}^{0}>-1$ holds, for all $t \geq 0$. For $\mathbb{Q} \in \mathcal{P}(\mathbb{G})$, by virtue of Girsanov's theorem, the process $W^{\mathbb{G}, \mathbb{Q}}=\left(W_{t}^{\mathbb{G}, \mathbb{Q}}\right)_{t \geq 0}$ defined by

$$
W_{t}^{\mathbb{G}, \mathbb{Q}}=W_{t}^{\mathbb{G}}-\int_{0}^{t} \mu_{s}^{\mathbb{G}} d s, \forall t \geq 0,
$$

is a $(\mathbb{Q}, \mathbb{G})$-standard Brownian motion and the process $M^{\mathbb{G}, \mathbb{Q}}=\left(M_{t}^{\mathbb{G}, \mathbb{Q}}\right)_{t \geq 0}$ defined by

$$
M_{t}^{\mathbb{G}, \mathbb{Q}}=M_{t}^{\mathbb{G}}-\int_{0}^{t \wedge \tau} \gamma_{s}^{0} \lambda_{s} d s=H_{t}^{\mathbb{G}}-\int_{0}^{t \wedge \tau}\left(1+\gamma_{s}^{0}\right) \lambda_{s} d s, \forall t \geq 0
$$

is a (uniformly integrable) $(\mathbb{Q}, \mathbb{G})$-martingale, where the process $\lambda$ is given by (4.9) above. The change of probability measure defined above changes the driving Brownian motion and the intensity rate of the random time $\tau$. The specific choice of $\gamma^{0}=0$ leads to a change of probability measure which does not affect the form of the intensity.

Remark 6.4. The set $\mathcal{P}(\mathbb{G})$ of equivalent martingale measures on $\mathbb{G}$ is strictly larger ${ }^{8}$ than $\mathcal{P}^{*}$, the set of $\mathbb{G}$-optional projections $L^{*, \mathbb{G}}$ of $L^{*}(\tau)$. In order to show this matter, we first note that any process $L^{*}(\tau)$ which is a Radon-Nikodym density of a measure $\mathbb{P}^{*}$ in $\mathcal{P}^{*}$ is given by $L^{*}(\tau)=L_{0}^{*}(\tau) K(\tau)$ with $L_{0}^{*}$ being a deterministic function and

$$
K_{t}(\tau)=\exp \left(\int_{0}^{t} \zeta_{s}(\tau) d W_{s}(\tau)-\frac{1}{2} \int_{0}^{t} \zeta_{s}^{2}(\tau) d s\right), \forall t \geq 0,
$$

where $K(\tau)$ is the same for all choices of $L^{*}(\tau)$. Therefore, the equality of $\mathcal{P}(\mathbb{G})$ and $\mathcal{P}^{*}$ would imply that any process of the form $\mathcal{E}\left(\mu^{\mathbb{G}} \cdot W^{\mathbb{G}}\right) \mathcal{E}\left(\gamma^{0} \cdot M^{\mathbb{G}}\right)$ can be written in the form $\mathcal{E}\left(\mu^{\mathbb{G}} \cdot W^{\mathbb{G}}\right) \mathcal{E}\left(\psi^{0} \cdot M^{\mathbb{G}}\right)$ with $\psi^{0}$ defined in (6.8). In other terms, for any $\mathbb{F}$-predictable process $\gamma^{0}=\left(\gamma_{t}^{0}\right)_{t \geq 0}$ such that $\gamma_{t}^{0}>-1$, for all $t \geq 0$, we are looking for a function $L_{0}^{*}$ such that

$$
\gamma_{t}^{0}=\frac{L_{0}^{*}(t) K_{t}(t)}{L_{t}^{*, 0}}-1, \forall t \geq 0 .
$$

We will prove that the existence of such a function $L_{0}^{*}$ is not possible for some specific choices of $\gamma^{0}$. The quantity $L_{t}^{*, 0}$ being computed in (4.6), the equation (6.9) is equivalent

\footnotetext{
${ }^{8}$ This can be explained by the fact that the multiplicity of the filtrations $\mathbb{F}^{(\tau)}$ and $\mathbb{F}$ is one, while the multiplicity of $\mathbb{G}$ is two (see [7] for the notion of multiplicity).
} 
to

$$
\begin{aligned}
G_{t} L_{0}^{*}(t) K_{t}(t) & =\left(1+\gamma_{t}^{0}\right) \int_{t}^{\infty} L_{t}^{*}(u) p_{t}(u) g(u) d u \\
& =\left(1+\gamma_{t}^{0}\right) \int_{t}^{\infty} L_{0}^{*}(u) K_{t}(u) p_{t}(u) g(u) d u, \forall t \geq 0 .
\end{aligned}
$$

Using the definition of $K(\tau)$ above, equality (4.2) and the form of $p(u)$ given in (3.2), one obtains

$$
K_{t}(u) p_{t}(u)=\exp \left(-\frac{\nu}{\rho} W_{t}-\frac{1}{2}\left(\frac{\nu}{\rho}\right)^{2} t\right), \forall t \geq 0
$$

so that this product does not depend on $u \geq 0$ and equality (6.10) can be rewritten as

$$
G_{t} L_{0}^{*}(t) K_{t}(t)=\left(1+\gamma_{t}^{0}\right) \exp \left(-\frac{\nu}{\rho} W_{t}-\frac{1}{2}\left(\frac{\nu}{\rho}\right)^{2} t\right) \int_{t}^{\infty} L_{0}^{*}(u) g(u) d u, \forall t \geq 0 .
$$

The choice of $\gamma_{t}^{0}=G_{t} K_{t}(t) \chi_{t}-1$, for all $t \geq 0$, for any strictly positive $\mathbb{F}$-adapted process $\chi=\left(\chi_{t}\right)_{t \geq 0}$ leads to the equality

$$
L_{0}^{*}(t)\left(\int_{t}^{\infty} L_{0}^{*}(u) g(u) d u\right)^{-1}=\chi_{t} \exp \left(-\frac{\nu}{\rho} W_{t}-\frac{1}{2}\left(\frac{\nu}{\rho}\right)^{2} t\right), \forall t \geq 0,
$$

which provides a contradiction to our assumptions above, since the left-hand side is deterministic, while the right-hand side is not, for $\nu \neq 0$. Note that, if $\nu=0$, then the stochastic exponential on the right-hand side above is equal to one. Hence, due to the continuity of the processes, we have $K(\tau)=1 / p(\tau)$, that corresponds to the choice of the decoupling measure from [2].

Remark 6.5. Note that one can assume from the beginning, without subsequent complications, that the process $S$ solves the stochastic differential equation

$$
d S_{t}=S_{t}\left(\nu_{t} d t+\rho_{t} d W_{t}\right), \quad S_{0}=1,
$$

where $\nu=\left(\nu_{t}\right)_{t \geq 0}$ and $\rho=\left(\rho_{t}\right)_{t \geq 0}$ as well as the interest rate are some appropriate F-adapted process, as soon as the appropriate model of financial markets is complete and arbitrage free. We can also extend the study above to the case where the interest rate is $\mathbb{G}$-adapted with $r_{t}(\tau)=\mathbb{1}_{\{\tau>t\}} r_{t}^{0}+\mathbb{1}_{\{\tau \leq t\}} r_{t}^{1}(\tau)$, for all $t \geq 0$, where the processes $r^{0}=\left(r_{t}^{0}\right)_{t \geq 0}$ and $r^{1}(u)=\left(r_{t}^{1}(u)\right)_{t \geq 0}, u \geq 0$, are $\mathbb{F}$-adapted.

\section{References}

[1] Aksamit, A. and Jeanblanc, M.: Enlargement of filtration with finance in view. Springer, Berlin, 2017. $\mathrm{x}+150$ pp. MR3729407

[2] Amendinger, J.: Initial Enlargement of Filtrations and additional information in financial markets. $\mathrm{PhD}$ thesis, Technical University Berlin.

[3] Amendinger, J.: Martingale representation theorems for initially enlarged filtrations. Stochastic Processes and their Applications 89, (2000), 101-116. MR1775229

[4] Bielecki, T. R., Jakubowski, J., Jeanblanc, M. and Niewęgłowski, M.: Special semimartingales and shrinkage of filtration. Annals of Applied Probability 31, (2021), 1376-1402. MR4278787

[5] Brémaud, P.: Point processes and queues: martingale dynamics. Springer, Berlin, 1981. xviii+354 pp. MR0636252

[6] Callegaro, G., Jeanblanc, M. and Zargari, B.: Carthaginian enlargement of filtrations. ESAIM: Probability and Statistics 17, (2013), 550-566. MR3085632

[7] Davis, M. H. A. and Varaiya, P.: The multiplicity of an increasing family of sigma-fields. Annals of Probability 2, (1974), 958-963. MR0370754 
Projections of martingales in enlargements of Brownian filtrations

[8] Dellacherie, C. and Meyer, P. A.: Probabilities and potential B. Theory of martingales. NorthHolland 82, Amsterdam, 1982. xvii+463 pp. MR0745449

[9] Duffie, D.: Stochastic equilibria: existence, spanning number, and the 'no expected financial gain from trade' hypothesis. Econometrica 54(5), (1986), 1161-1183. MR0859460

[10] El Karoui, N., Jeanblanc, M. and Jiao, Y.: What happens after a default: the conditional density approach. Stochastic Processes and their Applications 120, (2010), 1011-1032. MR2639736

[11] Elliott, R. J., Jeanblanc, M. and Yor, M.: On models of default risk. Mathematical Finance 10, (2000), 179-195. MR1802597

[12] Gapeev, P. V., Jeanblanc, M. and Wu, D.: Projections in enlargements of filtrations under Jacod's equivalence hypothesis for marked point processes. Preprint, hal-03207058, (2021).

[13] Grorud, A. and Pontier, M.: Insider trading in a continuous time market model. International Journal of Theoretical and Applied Finance 1, (1998), 331-347.

[14] He, S. W., Wang, J. G. and Yan, J. A.: Semimartingale theory and stochastic calculus. Science Press and CRC Press, Bocal Raton, 1992. xiv+546 pp. MR1219534

[15] Jacod, J.: Grossissement initial, hypothèse $\left(\mathrm{H}^{\prime}\right)$ et théorème de Girsanov. Lecture Notes in Mathematics, 1118, (1985), 15-35.

[16] Jacod, J. and Shiryaev, A. N.: Limit theorems for stochastic processes. Springer, Berlin, 1987. xviii+601 pp. MR0959133

[17] Jeanblanc, M., Yor, M. and Chesney, M.: Mathematical methods for financial markets. Springer, London, 2009. xxvi+732 pp. MR2568861

[18] Jeanblanc, M. and Song, S.: Martingale representation property in progressively enlarged filtrations. Stochastic Processes and their Applications 125(11), (2015), 4242-4271. MR3385602

[19] Jeanblanc, M. and Le Cam, Y.: Progressive enlargement of filtrations with initial times. Stochastic Processes and their Applications 119, (2009), 2523-2543. MR2532211

[20] Jeulin, Th.: Semi-martingales et grossissement d'une filtration. Lecture Notes in Mathematics 833, (1980), ix+142 pp. MR0604176

[21] Jeulin, Th. and Yor, M.: Inégalité de Hardy, semimartingales et faux-amis. Lecture Notes in Mathematics 721, (1979), 332-359. MR0544805

[22] Liptser, R. S. and Shiryaev, A. N.: Statistics of random processes I (second edition). Springer, Berlin, 2001. xvi+427 pp. MR1800857

[23] Protter, Ph. E.: Stochastic integration and differential equations (second edition, version 2.1). Springer, Berlin, 2005. xiv+419 pp. MR2273672

[24] Revuz, D. and Yor, M.: Continuous martingales and Brownian motion (third edition). Springer, Berlin, 1999. xiv+602 pp. MR1725357

[25] Song, S.: Optional splitting formula in a progressively enlarged filtration. ESAIM Probability and Statistics 18, (2014), 829-853. MR3334016

[26] Stricker, Ch. and Yor, M.: Calcul stochastique dépendant d'un paramètre. Zeitschrift für Wahrscheinlichkeitstheorie und verwandte Gebiete 45, (1978), 109-133. MR0510530

[27] Wu, D.: Density model and its application to credit counterparty risk. PhD thesis, Université d'Evry, 2013.

Acknowledgments. The authors thank warmly the anonymous referee for reading very carefully the two versions of the paper, pointing out many mistakes, misprints in the submitted versions. Following her/his questions and comments, and taking care about new references that she/he provided, we improved the quality of the paper. Monique Jeanblanc thanks Claudio Fontana and Marie-Claire Quenez for fruitful discussions. 